\title{
The Effect of Chemical Structure of OEG Ligand Shells with Quaternary Ammonium Moiety on the Colloidal Stabilization, Cellular Uptake and Photothermal Stability of Gold Nanorods
}

\author{
Sarka Salajkova, ${ }^{1,2}$ Filip Havel, ${ }^{1,3}$ \\ Michal Sramek, ' Filip Novotny, (D) 1,4 \\ David Malinak, (D) ${ }^{2,5}$ Rafael \\ Dolezal, (D) ${ }^{2,5}$ Lukas Prchal, $^{2}$ \\ Marketa Benkova, (D) ${ }^{2}$ Ondrej \\ Soukup, (D) ${ }^{2}$ Kamil Musilek, (DD 2,5 \\ Kamil Kuca, (D) ${ }^{2,5}$ Jiri Bartek, ${ }^{1,6,7}$ Jan \\ Proska, (1D ${ }^{3}$ Monika Zarska, (D) ${ }^{\prime}$ \\ Zdenek Hodny'
}

'Department of Genome Integrity, Institute of Molecular Genetics of the Czech Academy of Sciences, Prague, Czech Republic; ${ }^{2}$ Biomedical Research Center, University Hospital Hradec Kralove, Hradec Kralove, Czech Republic; ${ }^{3}$ Department of Physical Electronics, Faculty of Nuclear Sciences and Physical Engineering, Czech Technical University in Prague, Prague, Czech Republic; ${ }^{4}$ Center for Advanced Functional Nanorobots, Department of Inorganic Chemistry, Faculty of Chemical Technology, University of Chemistry and Technology Prague, Prague, Czech Republic; ${ }^{5}$ Department of Chemistry, Faculty of Science, University of Hradec Kralove, Hradec Kralove, Czech Republic; ' ${ }^{6}$ enome Integrity Unit, Danish Cancer Society Research Center, Copenhagen, Denmark; ${ }^{7}$ Department of Medical Biochemistry and Biophysics, Science for Life Laboratory, Division of Genome Biology, Karolinska Institute, Stockholm, Sweden

Correspondence: Monika Zarska

Department of Genome Integrity, Institute of Molecular Genetics of the Czech Academy of Sciences, Videnska 1083, Prague 4, Prague, CZ 142 20, Czech Republic

Tel +420- 24106 315I

Fax + 420-24106 2289

Email monika.zarska@img.cas.cz

Kamil Kuca

Biomedical Research Center, University Hospital Hradec Kralove, Sokolska 58I, Hradec Kralove, CZ 500 05, Czech Republic

Tel +420-495833447

Email kamil.kuca@fnhk.cz
Purpose: Plasmonic photothermal cancer therapy by gold nanorods (GNRs) emerges as a promising tool for cancer treatment. The goal of this study was to design cationic oligoethylene glycol (OEG) compounds varying in hydrophobicity and molecular electrostatic potential as ligand shells of GNRs. Three series of ligands with different length of OEG chain (ethylene glycol units $=3,4,5$ ) and variants of quaternary ammonium salts (QAS) as terminal functional group were synthesized and compared to a prototypical quaternary ammonium ligand with alkyl chain - (16-mercaptohexadecyl)trimethylammonium bromide (MTAB).

Methods: Step-by-step research approach starting with the preparation of compounds characterized by NMR and HRMS spectra, GNRs ligand exchange evaluation through characterization of cytotoxicity and GNRs cellular uptake was used. A method quantifying the reshaping of GNRs was applied to determine the effect of ligand structure on the heat transport from GNRs under fs-laser irradiation.

Results: Fourteen out of 18 synthesized OEG compounds successfully stabilized GNRs in the water. The colloidal stability of prepared GNRs in the cell culture medium decreased with the number of OEG units. In contrast, the cellular uptake of ${ }^{\mathrm{OEG}+}{ }^{+}$GNRs by HeLa cells increased with the length of OEG chain while the structure of the QAS group showed a minor role. Compared to MTAB, more hydrophilic OEG compounds exhibited nearly two order of magnitude lower cytotoxicity in free state and provided efficient cellular uptake of GNRs close to the level of MTAB. Regarding photothermal properties, OEG compounds evoked the photothermal reshaping of GNRs at lower peak fluence $\left(14.8 \mathrm{~mJ} / \mathrm{cm}^{2}\right)$ of femtosecond laser irradiation than the alkanethiol MTAB.

Conclusion: ${ }^{\mathrm{OEG}+} \mathrm{GNRs}$ appear to be optimal for clinical applications with systemic administration of NPs not-requiring irradiation at high laser intensity such as drug delivery and photothermal therapy inducing apoptosis.

Keywords: gold nanorods, quaternary ammonium salts, oligoethylene glycol, cellular uptake, photothermal stability

\section{Introduction}

Colloidal gold nanoparticles (GNPs) have been studied in a wide range of research areas attributable to their specific optical properties which originate from the interaction of light with their free conduction electrons, known as localized surface plasmon resonance (LSPR). ${ }^{1-3}$ Especially gold nanorods 
(GNRs) have attracted particular attention due to their two distinctive extinction bands that are associated with transverse and longitudinal modes of LSPR. Optical response can be further controlled by tuning the particle size, shape, composition, and dielectric constants of the particle material as well as of the surrounding medium. ${ }^{4-6}$ Under light irradiation, the LSRP of GNRs leads to partial conversion of absorbed light into heat. In such a scenario, gold nanoparticles act as nanosized sources of heat. ${ }^{7}$ The described phenomenon attracts attention of scientists for its promising applications in biomedical fields such as plasmonic photothermal cancer therapy, ${ }^{8}$ controlled drug delivery and release, ${ }^{9}$ and microtissue surgery. ${ }^{10}$

Gold nanoshells stabilized by polyethylene glycol (PEG) were demonstrated as the first plasmonic nanoparticle-based agents for photothermal therapy of a cancer in clinical trials based on their accumulation in tumor ascribed to the enhanced permeability and retention effect. $^{11}$ Ethylene glycols are particularly promising ligands of GNPs for their minimal toxicity, increased polarizability, minimal interaction with biomacromolecules and ability to escape opsonization, the latter preventing recognition of GNPs by immune system. ${ }^{12-17}$ Moreover, PEG increases the stability and solubility of the NPs under physiological conditions by increasing the steric distance between NPs that prevents their aggregation and increasing hydrophilicity of NPs due to strong surface hydration of ethylene glycol units. ${ }^{18-20}$ Although the PEG coatings improve the pharmacokinetics of NPs and increase the blood circulation time, ${ }^{21,22}$ they also hinder the desired nanoparticle uptake by cancer cells. It is known that the cellular uptake of neutral NPs, including uncharged PEGylated NPs, is much lower in comparison to NPs with positively and/or negatively charged surfaces. ${ }^{23-25}$ Additionally, the rod-like shape of NPs can significantly reduce the uptake of NPs by, for example, macrophages. $^{26,27}$ These both limit the utilization of PEGylated gold nanorods in applications that require high incorporation of NPs into cells such as cell transfection $^{28,29}$ and photothermal cancer therapy utilizing the tumor tropic properties of stem cells to deliver NPs to the tumor area. ${ }^{30-32}$

Depending on the light excitation, one can trigger a variety of photothermally induced effects, namely nanoparticle fragmentation, ${ }^{33}$ nanoparticle reshaping, ${ }^{34}$ microbubble generation, ${ }^{35,36}$ or generation of acoustic and shock waves $^{37}$ that may occur during the photothermal ablation of cancer cells. Several studies reveal underlying mechanisms of the photothermal conversion consisting of a sequence of events, namely the plasmon excitation, electron-phonon thermalization, and heat diffusion. ${ }^{7,38}$ Recent advances in the thermoplasmonics propose both theoretically and experimentally that the solvation layer of nanoparticles may considerably influence the heat transfer from the nanoparticle to its surroundings, especially for the case of pulsed illumination. ${ }^{39-44}$ Horiguchi et $\mathrm{al}^{42}$ showed that the photothermal reshaping of GNRs depends on the type of their surface modification when nanosecond pulsed laser irradiation induced larger spectral changes of PEG-GNRs and phosphatidylcholine (PC)-passivated GNRs than in the case of poly(ethylenesulfonate hydrochloride) (PSS)- and poly(vinylpyrrolidone) (PVP)-passivated GNRs. Similarly, a method of transient absorption spectroscopy revealed that the thermal dissipation from photoexcited GNRs depends on the ligand chemistry and on the ligand ability to exclude water from the surface of the GNRs that makes the phonon transport between the GNRs and solvent less efficient. ${ }^{43}$ The role of ligand hydrophilicity was confirmed by $\mathrm{Wu}$ et al, ${ }^{44}$ who demonstrated that the effective thermal conductance of the GNRs/surfactant layer/water system is higher for GNRs modified by hydrophilic PEG than for GNRs modified by hydrophobic cetyltrimethylammonium bromide (CTAB) with the same length of surfactant layer. Possibly the covalent bonding between $\mathrm{Au}$ and thiol-containing PEG and water penetration into the PEG layer are the reasons of enhanced heat dissipation. In addition, Centi et $\mathrm{al}^{45}$ found that relatively small thiols (methylbenzenethiol) dramatically enhance the thermal stability of GNRs during annealing in an oven set to $90^{\circ} \mathrm{C}$ and their photostability during photoacoustic monitoring compared to PEG (MW 5,000 Da).

Recently, we developed the GNRs stabilized by surfactant composed of the short ethylene glycol chain with thiol moiety and quaternary ammonium head (trimethylammonium) as terminal functional group - $N, N$, $N$-trimethyl-3,6,9,12,15-pentaoxaheptadecyl-17-sulfanyl1 -ammonium bromide (POSAB). ${ }^{46}$ While the polar ethylene glycol chain strongly reduced the compound cytotoxicity in free state compared to the common alkyl chain, quaternary ammonium salt (QAS) provided GNRs high cellular uptake. In connection with these results here we investigated the role of different length of oligoethylene glycol (OEG) chain and various structure of quaternary ammonium head on the physical- 
chemical and biological properties of free compound as well as ligand shell of GNRs. Three series of ligands with 3, 4 and 5 ethylene glycol units and six variants of quaternary ammonium heads were rationally designed and synthesized. Their ability to ensure the colloidal stability of GNRs, the rate of GNRs cellular uptake and cytotoxicity of GNRs as well as free ligands were evaluated. Finally, the effect of solvation layer on GNRs thermal stability under the femtosecond (fs) laser irradiation was measured for the representative OEG ligands showing high cellular uptake and good stability and was compared to a known alkyl analogue (16-mercaptohexadecyl)trimethylammonium bromide; MTAB) ${ }^{47,48}$

\section{Materials and Methods}

\section{Chemicals and Antibodies}

3-(4,5-dimethylthiazol-2-yl)-2,5-diphenyltetrazolium

bromide (MTT), 4',6-diamidino-2-phenylindole (DAPI, D9542), ascorbic acid, bovine serum albumin (BSA), hydrogen tetrachloroaurate(III) trihydrate $\left(\mathrm{HAuCl}_{4}\right.$. $3 \mathrm{H}_{2} \mathrm{O}$ ), cetyltrimethylammonium bromide $(\mathrm{CTAB})$, silver nitrate $\left(\mathrm{AgNO}_{3}\right)$ and sodium borohydride $\left(\mathrm{NaBH}_{4}\right)$ were purchased from Sigma-Aldrich (St. Louis, MO, USA). Antibody against lysosome-associated membrane protein 1 (LAMP-1; sc-20011) was from Santa Cruz Biotechnology (Dallas, TX, USA). Alexa Fluor 488 phalloidin (A12379), Alexa Fluor 488-conjugated secondary antibody and ProLong Gold Antifade Mountant were from Invitrogen (Carlsbad, CA, USA). Milli-Q water $\left(18.2 \mathrm{M} \Omega\right.$ at $\left.25^{\circ} \mathrm{C}\right)$ used for nanoparticle synthesis was from Ardeapharma a. s., Sevetin, Czech Republic.

\section{Cell Cultures}

Human cervical carcinoma cell line HeLa obtained from ATCC (Manassas, VA, USA) was cultured in Dulbecco's Modified Eagle's Medium (DMEM; Biochrom AG, Berlin, Germany; containing $4.5 \mathrm{~g} / \mathrm{L}$ glucose) supplemented with 10\% Fetal Bovine Serum (FBS; Gibco; Grand Island, NY, USA). Chinese hamster ovary cell line CHOK1 from ECACC (Salisbury, UK) was cultured in Nutrient Mixture F-12 Ham (Sigma-Aldrich) growth medium supplemented with 10\% FBS (Sigma-Aldrich). Both culture media were supplemented with penicillin $(100 \mathrm{U} / \mathrm{mL})$ and streptomycin $(100 \mu \mathrm{g} / \mathrm{mL}$; SigmaAldrich). Cells were cultivated at $37^{\circ} \mathrm{C}$ under $5 \% \mathrm{CO}_{2}$ atmosphere and $95 \%$ humidity.
Experimental Evaluation and Calculation of the Hydrophobicity of Prepared Ligands

Hydrophobicity of final compounds expressed as $\mathrm{C} \log P$ was calculated in Open Babel, version 2.3.1 (http://openbabel.org, accessed October 2011). The correlation with experimental evaluation of hydrophobicity by high performance liquid chromatography (HPLC) with isocratic elution $(\log k)$ is described in the Supplementary Material.

\section{Calculation of Molecular Electrostatic Potential (ESP)}

The models of prepared compounds were pre-designed in HyperChem 8.0 software (Hypercube, Gainesville, FL, USA) as free cations and exported as mol files for further calculations. In Spartan 14 (Wavefunction, Irvine, CA, USA), semi-empirical quantum chemistry PM6 method was used for determination of the compound equilibrium geometry in vacuum. Electrostatic potential of the geometrical optimized conformers in the local minimum of potential energy was mapped on the electron isodensity surface of $0.002 \mathrm{e} / \mathrm{b}^{3}$. The atomic partial charges were determined on the same level of theory in Spartan 14 applying CHELP algorithm for a least-square fit of the partial charges to the molecular electrostatic potential (ie, ESP atomic partial charges). Additional computational models obtained by molecular dynamic based conformational analyses with the semi-empirical method RM1 are described in the Supplementary Material.

\section{Cell Viability Assessment of Ligands in Free State}

In order to compare the cytotoxic effect of the studied compounds standard MTT assay (3-(4,5-dimethylthiazol2-yl)-2,5-diphenyltetrazolium bromide) was utilized according to the manufacturer's protocol using the CHOK1 cells. ${ }^{49}$ The protocol is described in detail in the Supplementary Material.

\section{Preparation of Cetyltrimethylammonium Bromide (CTAB)-Stabilized Gold Nanorods and Their Functionalization by OEG Compounds}

Gold nanorods (GNRs) were prepared by a modification of the seeded-growth method in the presence of silver nitrate 
according to the protocols described previously. ${ }^{48,50,51}$ The protocol is described in detail in the Supplementary Material.

To modify GNRs by OEG compounds and by MTAB, CTAB-coated GNRs $(5.7 \mathrm{~mL}$ of GNRs tuned to $633 \mathrm{~nm}$ or $7.0 \mathrm{~mL}$ of GNRs tuned to NIR) were purified by two cycles of centrifugation $(4,700 \times \mathrm{g}$ for GNRs tuned to 633 $\mathrm{nm}$ or $7,400 \times \mathrm{g}$ for GNRs tuned to NIR for 20 minutes), redispersation of sedimented GNRs in the Milli-Q water and concentration to $1 \mathrm{~mL}$ (residual $\mathrm{CTAB}$ in solution was $\leq 0.5 \mathrm{mM}$ ). After that, $1 \mathrm{~mL}$ of $5 \mathrm{mM}$ aqueous solution of MTAB or cationic OEG compounds was directly added to the concentrated GNRs dispersion and kept at room temperature for a minimum of 7 days. Next, the GNRs solution was diluted by Milli-Q water $(7 \mathrm{~mL})$ and sonicated at $85 \mathrm{~W}$ and $45^{\circ} \mathrm{C}$ for 2 hours. Afterwards, the residual unconjugated ligand was removed by two cycles of centrifugation in the following 2 days. The final concentration of prepared GNRs ranged from 0.35 $\mathrm{mM}$ to $0.45 \mathrm{mM}$ of gold atoms $\left(\mathrm{Au}^{0}\right)$ in the nanoparticle dispersion as calculated by formula $\mathrm{Au}^{0}[\mathrm{mM}]=0.355 \times$ $\mathrm{A}_{390 \mathrm{~nm}} /$ path length $[\mathrm{cm}]{ }^{48,52}$ To assess the colloidal stability of GNRs, the UV-VIS-NIR absorption spectra of GNRs dispersions were measured by a DU 730 Life Science UV/VIS Spectrophotometer (Beckman Coulter, Fullerton, CA, USA).

\section{Quantification of Ligand Packing Density by Inductively Coupled Plasma-Optical Emission Spectrometry (ICP-OES)}

To quantify the ligand packing density, the GNRs functionalized by cationic ligands were analyzed by ICP-OES (Spectro Arcos MV, Spectro Analytical Instruments, Kleve, Germany; see the Supplementary Material for detailed measurement conditions). The ligand packing density on GNRs was then calculated from the ratio of the mass fraction of sulfur (S) and gold (Au) as described previously. ${ }^{53,54}$ The parameters of GNRs were estimated from FE-SEM size analysis of $300 \mathrm{NPs}$, the calculation is shown in the Supplementary Material. The packing density $\sigma_{\text {calc. }}$ was calculated by the equation:

$$
\sigma_{\text {calc }}=w_{S} \frac{1}{w_{A u}} N_{A} \rho_{G N R s} V_{G N R s} \frac{1}{M_{S}} \frac{1}{S_{G N R S}}
$$

where $w_{A u}$ and $w_{S}$ are the $\mathrm{Au}$ and $\mathrm{S}$ mass fractions determined using ICP-OES, $N_{A}$ is the Avogadro constant, $\rho_{\text {GNRS }}$ is the density of gold, $M_{S}$ is the molar mass of $S, V_{G N R s}$ and $S_{G N R s}$ are the volume and surface of GNRs, respectively. Data were expressed as a mean of two independent functionalizations of GNRs measured in the triplicate.

\section{Cellular Uptake of GNRs Determined by Confocal Microscopy and}

\section{Fluorescence-Activated Cell Sorting (FACS)}

HeLa cells were seeded onto coverslips or 6-well plates $\left(4 \times 10^{4}\right.$ cells per coverslip and $3 \times 10^{5}$ cells per well $)$ and allowed to attach overnight (16 hours). Then the cells were incubated with ${ }^{\mathrm{MTAB}} \mathrm{GNRs}$ or ${ }^{\mathrm{OEG}+}{ }^{+} \mathrm{GNRs}$ at a concentration $20 \mu \mathrm{M}\left(\mathrm{Au}^{0}\right)$ diluted into serum-containing cell culture medium for 24 hours. Cells cultured in nanoparticle-free media were used as controls.

For microscopic analysis, the cells were washed $3 \times$ with phosphate buffered saline (PBS), fixed with $4 \%$ formaldehyde (15 minutes, r.t.) and permeabilized with $0.2 \%$ Triton X-100 (10 minutes). Washed cells were blocked with 3\% BSA (30 minutes) and incubated with Alexa Fluor 488-conjugated phalloidin for $\mathrm{F}$-actin staining (diluted 1:200 in $\mathrm{PBS} / 3 \% \mathrm{BSA} ; 37^{\circ} \mathrm{C}$; 1 hour in dark) or with anti-LAMP-1 primary antibody (diluted 1:250 in PBS $/ 0.1 \%$ Tween-20; 1 hour), washed and stained by Alexa 488-conjugated secondary antibody (diluted $1: 1,000$ in PBS $/ 0.1 \%$ Tween-20; 1 hour in the dark). After the final wash, the nuclei were stained by 4',6-diamidino-2-phenylindole (DAPI; $1 \mu \mathrm{g} / \mathrm{mL}$ ) and coverslips were mounted with the ProLong mounting medium. The fluorescence and back-scattered laser light from the longitudinal LSPR mode of GNRs was detected with Leica TCS SP8 AOBS confocal microscope (Leica Microsystems, Wetzlar, Germany) using the following excitation and detection wavelengths: DAPI (405/413 $488 \mathrm{~nm})$, Alexa Fluor $488(498 / 510-560 \mathrm{~nm})$ and GNRs $(633 / 630-636 \mathrm{~nm})$ as described previously. ${ }^{48}$

For FACS analysis, the cells in 6-well plates were washed $3 \times$ with PBS, trypsinized with $0.25 \%$ trypsin/ $0.53 \mathrm{mM}$ EDTA and after centrifugation $(700 \times \mathrm{g}$; $3 \mathrm{~min}-$ utes) fixed with $4 \%$ formaldehyde ( 15 minutes, r.t.). The cells were counted by BD LSR II (BD Biosciences, San Jose, CA, USA). The scattered light of the longitudinal LSPR mode of GNRs was detected using $633 \mathrm{~nm}$ laser in the side scatter mode of the cytometer at $635 \pm 10 \mathrm{~nm}$, as described previously. ${ }^{48}$ Data were expressed as a mean value and SD of triplicate experiments. 


\section{Evaluation of the Plasmonic Photothermal Stability of GNRs}

${ }^{\mathrm{MTAB}}$ GNRs or ${ }^{\mathrm{OEG}+}$ GNRs were deposited on the formvar coated TEM locator grid. The extra thick layer of formvar was resistant to applied fs-laser irradiation, the locator grid allowed to characterize the same area of the sample before and after the laser exposition on FE-SEM. Desired distribution of GNRs on the TEM grid was accomplished by depositing a droplet of GNRs solution of known concentration and subsequent control of the deposition time and air humidity. After the deposition process, TEM grids were carefully washed in ultrapure water and imaged on JSM$7500 f$ JEOL FE-SEM. Laser exposition was performed on an upright two-photon excitation microscope (2PEM, Bruker Ultima IntraVital, Middleton, WI, USA; equipped with Coherent Chameleon Ultra I laser, Santa Clara, CA, USA) using the following layered setup: microscope slide - TEM grid - water droplet - cover glass - water droplet - objective $(25 \times$ water immersion objective with a 1.1 numerical aperture). Samples were exposed to $140 \mathrm{fs}$ laser pulses at $750 \mathrm{~nm}$ with a repetition rate of $80 \mathrm{MHz}$ and peak fluence ranging from $14.8 \mathrm{~mJ} / \mathrm{cm}^{2}$ to $148.5 \mathrm{~mJ} /$ $\mathrm{cm}^{2}$. After the laser irradiation, the TEM grid was left to dry out and the selected areas were again characterized on FE-SEM. The statistical data from more than 300 nanoparticles were extracted by software analysis of acquired micrographs.

\section{Statistical Analysis}

Student's $t$-test was used to determine the statistical significance of arithmetic means' differences among groups: not significant (ns), $P<0.05 \quad(*), \quad P<0.01 \quad(* *)$, and $P<0.001$ (***).

\section{Results}

\section{Organic Synthesis}

The preparation of monoquaternary thiol with OEG side chain was based on commercially available symmetrical diols 1-3 with required length of chain (Figure 1A; for details see Supplementary Figure S1). Briefly, the desymmetrization reaction of two chemically equivalent hydroxyl groups was made in the first step by the introduction of

A
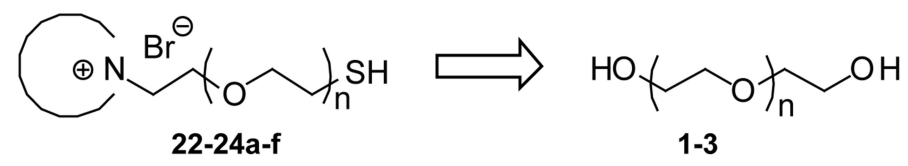

1-3

B

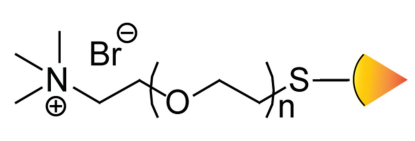

22-24a

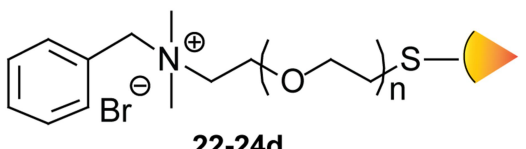

22-24d

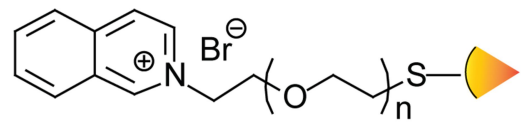

22-24e

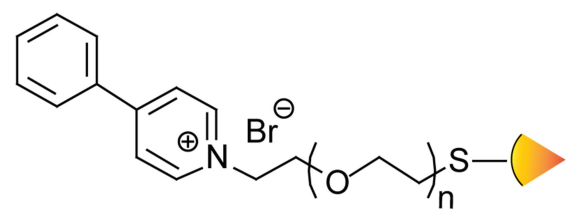

22-24c

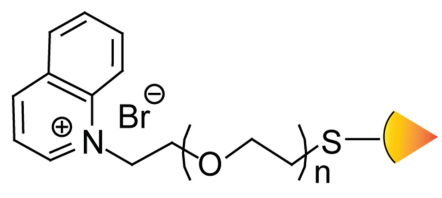

22-24f
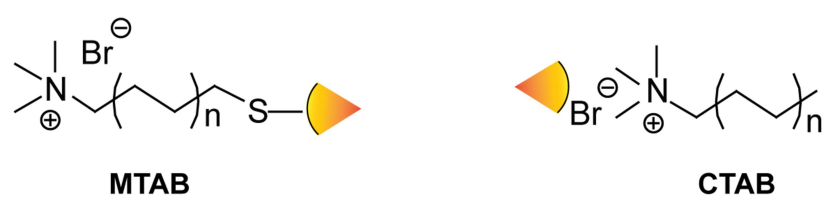

22: $n=3$

23: $n=4$

24: $n=5$

MTAB, CTAB: $n=7$

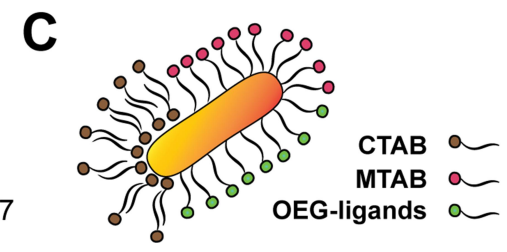

Figure I Retrosynthesis and structure of prepared cationic compounds for functionalization of GNRs (n, number of ethylene glycol units). (A) Retrosynthetic scheme of preparation quaternary ammonium compounds based on oligoethylene glycol chain. (B) Chemical structures of utilized compounds 22-24a-f and MTAB tethered to the surface of GNRs via S-Au bond and of CTAB non-covalently bonded to the GNRs surface. (C) Scheme of CTAB bilayer compared to monolayer of MTAB and OEG-ligands stabilizing the GNRs. 
the dimethoxytrityl group, which provided monoprotected alcohol intermediates 4-6. The subsequent step was the tosylation of the remaining free hydroxyl with following nucleophilic substitution of the tosyl group with potassium thioacetate to obtain intermediates 10-12. Further, thioacetates 13-15 were prepared by selective deprotection of dimethoxytrityl under acidic condition and consequent brominated thioacetates 16-18 via formation of triphenylphosphonium salt and following nucleophilic substitution. The quaternization of brominated thioacetates 16-18 were made by Menshutkin-like reaction with appropriate amine leading to quaternary thioacetates 19-21a-f. Deprotection of the thiol group was carried out in a mixture of anhydrous solvents (methanol, dichloromethane) by in situ generated hydrogen chloride. Synthesized QAS were obtained in $35-53 \%$ overall yields for final products 22-24ae (Supplementary Table S1) after seven steps. For quinolinium based QAS (22-24f) the overall yields were 15-28\%. The ligands were grouped into four series according to their side chain: ligands with ethylene glycol chain with different number of units, namely with three $\left(\mathrm{OEG}_{3}\right.$ series; cpd. 22a-f), four $\left(\mathrm{OEG}_{4}\right.$ series; cpd. 23a-f), and five $\left(\mathrm{OEG}_{5}\right.$ series; cpd. 24a-f) ethylene glycol units and the ligand with alkyl chain (MTAB) (Figure 1B and $\mathrm{C}$ ).

\section{Hydrophobicity and Molecular Electrostatic Potential of Prepared Compounds}

As the organic compounds are an important constituent part of the ligand-GNRs complex that can modify nanoparticle-cell interactions, cytotoxicity of NPs and their health-related applications, ${ }^{55-57}$ several theoretical and experimental molecular descriptors including the hydrophobicity and molecular electrostatic potential (ESP) of free compounds were assessed. Hydrophobicity supports transmembrane penetration, while the hydrophilicity provides the solubility in aqueous environment. ${ }^{58-60} \mathrm{In}$ addition, cytotoxicity of QAS is closely related to their hydrophobicity and especially the ability to incorporate the side chain of QAS compounds into phospholipid bilayer membrane. ${ }^{61,62}$ The hydrophobicity of final products was expressed as $\mathrm{C} \log P$ calculated in Open Babel 2.3.2 software. The relationship between $C \log P$ and cytotoxic potential of compounds is shown in Figure 2A. The calculated data proved that the compound with an alkyl chain (MTAB) and its analogue without thiol moiety (CTAB) are more hydrophobic than compounds with OEG chain (see Supplementary Table S1 for values of $(\log P$ ). Moreover, the correlation of Clog $P$ with experimental evaluation of hydrophobicity expressed as $\log k^{63}$ is described in detail in the Supplementary Material (see Supplementary Figure $\underline{\mathrm{S} 2 \mathrm{~A}}$ ). For OEG compounds, the hydrophobicity slightly increased with the length of OEG chains. Based on different structures of the cation, the compounds with trimethylammonium head (22-24a) followed by pyridinium salts (22-24b) showed the lowest hydrophobicity. Increased and comparable hydrophobicity was found for quinolinium (22-24f), isoquinolinium (22-24e), and benzalkonium (22-24d) salts. The most hydrophobic compounds were the phenylpyridinium salts $(\mathbf{2 2 - 2 4 c})$.

The interaction of NPs with proteins, the rate of their cellular uptake as well as their organ and sub-organ distribution strongly depend on the surface charge of NPs. ${ }^{24,64,65}$ Therefore, the ESP mapped on the frontier electron isodensity surface (ie, $0.002 \mathrm{e} / \mathrm{b}^{3}$, covering $99.1 \%$ of the total electron density) and the ESP atomic partial charge projected on the atomic nucleus on isodensity surface of the quaternary nitrogen were calculated in Spartan 14 software by semi-empirical quantum chemistry PM6 methods (see Figure 2B for selected compounds, Supplementary Table S1 and Supplementary Figure S3A for all final products and Supplementary Figure S3B for additional computational models obtained by conformational analyses on the semi-empirical method RM1). ${ }^{61,63}$ As expected, the OEG chain exhibited higher fluctuation of ESP than the alkyl chain due to the presence of oxygen, which caused variation in electron distribution along the chain. However, the disparity between the OEG chain with a different number of ethylene glycol units (22a and 24a) and alkyl chain (MTAB) did not exhibit a significant influence on the charge of quaternary nitrogen. In contrast, the introduction of quaternary ammonium nitrogen in the heteroaromatic system or solely aliphatic substitution exhibited substantial influence on the nitrogen ESP partial charge. Apparently, the nitrogen with the methyl substituent, namely trimethylammonium (24a) and benzalkonium (24d) salt, exhibited the highest partial ESP charge due to the electron-donating effect of the methyl group. In the case of compounds with quaternary nitrogen in the aromatic system, the partial charge was distributed through the aromatic ring. 
A

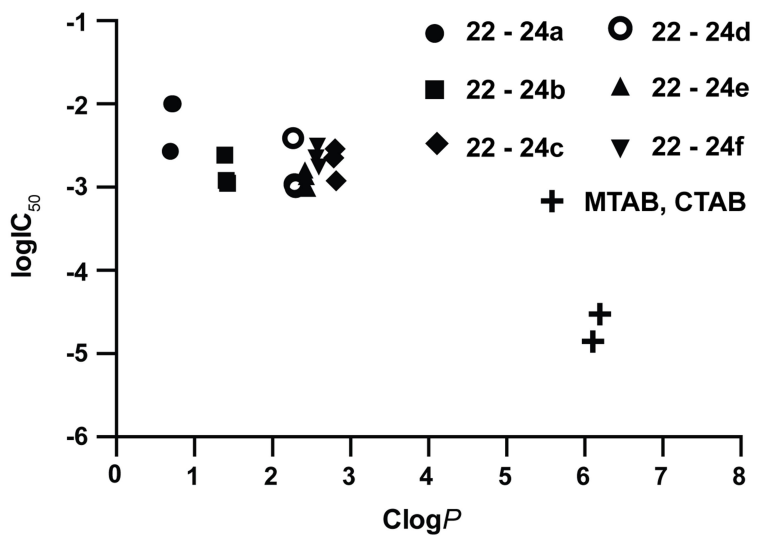

B
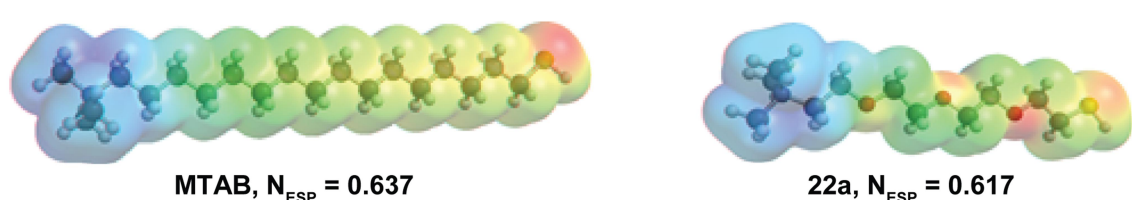

MTAB, $\mathrm{N}_{\mathrm{ESP}}=\mathbf{0 . 6 3 7}$

22a, $\mathrm{N}_{\mathrm{ESP}}=0.617$
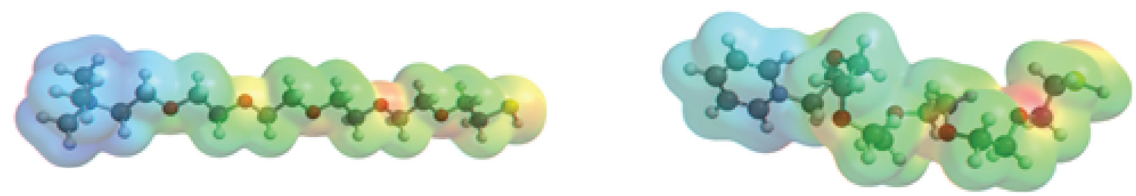

$24 \mathrm{a}, \mathrm{N}_{\mathrm{ESP}}=0.617$

$24 \mathrm{~b}, \mathrm{~N}_{\mathrm{ESP}}=0.433$
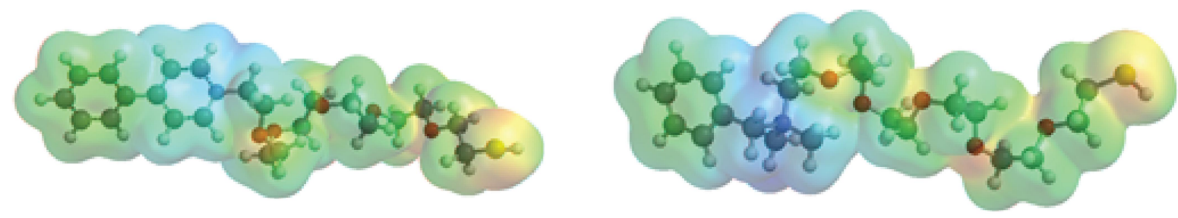

$24 \mathrm{C}, \mathrm{N}_{\mathrm{ESP}}=0.387$

24d, $N_{E S P}=0.738$
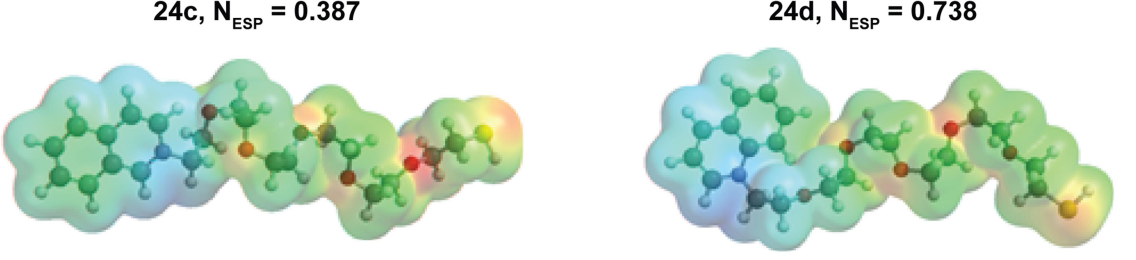

$24 \mathrm{e}, \mathrm{N}_{\mathrm{ESP}}=0.359$

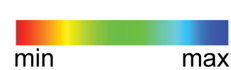

$24 f, N_{E S P}=0.164$

Figure 2 Hydrophobicity, models of molecular electrostatic potential and cytotoxicity of prepared compounds in free state. (A) Correlation between cytotoxicity (loglC 50 ) and calculated hydrophobicity (ClogP) of cationic compounds with OEG chain (22-24a-f) and alkyl chain (MTAB, CTAB). (B) Electrostatic potential maps and ESP atomic partial charges on the quaternary nitrogen $\left(\mathrm{N}_{\mathrm{ESP}}\right)$ of selected compounds determined in Spartan 14 molecular modeling and computational chemistry software.

\section{Functionalization of GNRs by OEG Compounds and Evaluation of Colloidal Stability and Ligand Packing Density of Cationic GNRs}

The GNRs coated by novel ligand shell $\left({ }^{\mathrm{OEG}+} \mathrm{GNRs}\right)$ were prepared by a ligand exchange of ${ }^{\mathrm{CTAB}} \mathrm{GNRs}(56.3 \pm 4.9 \mathrm{~nm}$ in length and $28.2 \pm 3.9 \mathrm{~nm}$ in width; see Supplementary Figure S4A and $\underline{\mathrm{C}}$ for size distribution of GNRs and their UV-Vis-NIR spectra, respectively) according to our protocol published previously. ${ }^{46,48}$ The presence of silver, which is a common reagent used in synthesis of GNRs, in ${ }^{\mathrm{OEG}+}$ GNRs was measured by elementary analysis (ICPOES) and was approx. $2 \%(\mathrm{Ag} / \mathrm{Au})$. The dimensions of GNRs were designed to obtain the nanorods with light absorption in the red region of VIS $(633 \mathrm{~nm})$ to enable visualization and determination of their behavior in living cells by commonly available equipment (confocal microscopes and fluorescence-activated cell sorter). The functionalization of GNRs by cationic OEG ligands allowed to 
prepare stable colloidal GNRs without affecting their shape, as was confirmed by measurements of UV-VISNIR absorption spectra. The typical absorption spectrum of GNRs consisted of the transverse $\left(\lambda_{\max } \sim 520 \mathrm{~nm}\right)$ and longitudinal $\left(\lambda_{\max } \sim 610-630 \mathrm{~nm}\right)$ absorption peaks (Figure 3A). Nevertheless, the ligand exchange was not successful for ligands 23a, 23c, 23f, and 24f. In this case, UV-VIS-NIR absorption spectra measured after functionalization of GNRs showed that GNRs tend to aggregate together as demonstrated by extensive change of the GNRs absorption spectrum. The decrease in the ratio of longitudinal and transverse absorption peak intensity accompanied by duplication/broadening and shifting of the longitudinal peak to longer wavelengths was observed. ${ }^{23 a, c, f}$ GNRs and ${ }^{24 f}$ GNRs were thus excluded from further biological evaluation.

The good colloidal stability of GNRs in biological media containing salts, low molecular compounds and biopolymers is a prerequisite for their further biological utilization. Therefore, the ability of cationic OEG ligands to stabilize GNRs in colloidal solution was assessed in the cell culture medium. The water stable ${ }^{\mathrm{OEG}+} \mathrm{GNRs}$ preparations were diluted to DMEM supplemented with $10 \%$ FBS (Figure 3B). Except MTAB analogue ligand 22a that underwent aggregation, the GNRs modified by $\mathrm{OEG}_{3}$ series showed similar colloidal stability in cell culture media as in water solution. In contrast, the $\mathrm{OEG}_{4}$ and $\mathrm{OEG}_{5}$ series provided less stable colloidal nanoparticles as indicated by changes of GNRs absorption spectra in most cases from slight reshaping of absorption peaks up to the fusion of transverse and longitudinal peaks and their extension to longer wavelengths demonstrating extensive GNRs aggregation (namely ligands $\mathbf{2 3 b}$ in $\mathrm{OEG}_{4}$ and $\mathbf{2 4 a}$ and $\mathbf{2 4 b}$ in $\mathrm{OEG}_{5}$ series). The colloidal stability of cationic GNRs according to the side chain of compounds decreased in the following order: MTAB alkyl chain $>\mathrm{OEG}_{3}$ chain $>$ $\mathrm{OEG}_{4} / \mathrm{OEG}_{5}$ chains (for absorption spectra of ${ }^{\mathrm{MTAB}} \mathrm{GNRs}$, see Supplementary Figure S5A). According to the divergent structure of cationic heads, the best colloidal stability was obtained for the benzalkonium (d) and isoquinolinium (e) ligands that showed in all tested lengths of OEG chain a sufficient stability demonstrated by a stable spectrum in the cell culture medium $\left(\mathrm{OEG}_{3}\right.$ and $\mathrm{OEG}_{5}$ series for quaternary ammonium head $\mathbf{d} ; \mathrm{OEG}_{3}$ and $\mathrm{OEG}_{4}$ series for e) or the altered spectrum still consisting of two distinctive plasmonic peaks $\left(\mathrm{OEG}_{4}\right.$ series for $\mathbf{d} ; \mathrm{OEG}_{5}$ for $\left.\mathbf{e}\right)$.

The packing density of OEG-coated GNRs was calculated based on the ratio of the mass fraction of sulfur (S) and gold $\mathrm{(Au})$ measured simultaneously by ICP-OES, ${ }^{53}$ where the gold-to-sulfur ratio depends proportionally on the volume-to-surface area ratio. ${ }^{54}$ Assuming complete removal of free ligand by centrifugation during sample preparation, the ligand coverage was estimated for all ${ }^{\text {OEG }}{ }^{+}$GNRs, including unstable colloidal dispersion of GNRs (23a, 23c, 23f, and 24f; Figure 3C). Importantly, the ligands that did not allow us to prepare stable GNRs in colloidal solution after the ligand exchange, reached less than half the ligand packing density compared to MTAB providing the highly stable colloid of GNRs. The different length of OEG chain did not have a significant influence on coverage of GNRs. However, the different packing density was conspicuous in comparison of each terminal cationic group. The more effective GNRs coverage exhibited the OEG ligands with benzalkonium salt (d) that in the combination with five ethylene glycol units $\left(\mathrm{OEG}_{5}\right.$; 24d) reached a similar packing density $(6.10 \pm 1.04$ molecules $\left./ \mathrm{nm}^{2}\right)$ to MTAB $\left(6.30 \pm 0.72\right.$ molecules $\left./ \mathrm{nm}^{2}\right)$.

\section{Assessment of Cytotoxicity of Cationic Ligands in Free State and in Complex with GNRs}

Previously, we showed that the cationic alkanethiol(MTAB)-stabilized GNRs are non-toxic both in vitro and in vivo. ${ }^{66}$ Similarly, no significant decrease in viability of $\mathrm{HeLa}$ cells was observed for all ${ }^{\mathrm{OEG}}{ }^{+}$GNRs tested at a concentration range from $30-50 \quad \mu \mathrm{M} \quad\left(\mathrm{Au}^{0}\right)$ (Supplementary Figure S6). However, the possibility of ligand release from the surface of GNRs motivated us to evaluate the cytotoxicity of free compounds by colorimetric MTT assay as a half maximal inhibitory concentration $\left(\mathrm{IC}_{50}\right){ }^{67} \mathrm{CHO}-\mathrm{K} 1$ cells previously established for the evaluation of cellular toxicity of $\mathrm{QAS}^{63,68}$ were used for the cell viability assay. As we showed recently, ${ }^{46}$ the compound with alkyl chain (MTAB) showed more than two orders of magnitude higher cytotoxicity than its ana$\log$ with OEG chain of similar length (24a). This correlated with higher hydrophobicity of alkanethiols compared to the OEG compound (see Figure 2A and Supplementary Table S1). Comparing the cytotoxicity of each quaternary ammonium head, compounds containing trimethylammonium group exhibited in the chain with four and five ethylene glycol units (23-24a) almost one order of magnitude lower in cytotoxicity compared to other OEG compounds (Supplementary Table S1). Additionally, the increasing length of chain of the 


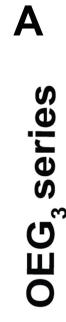
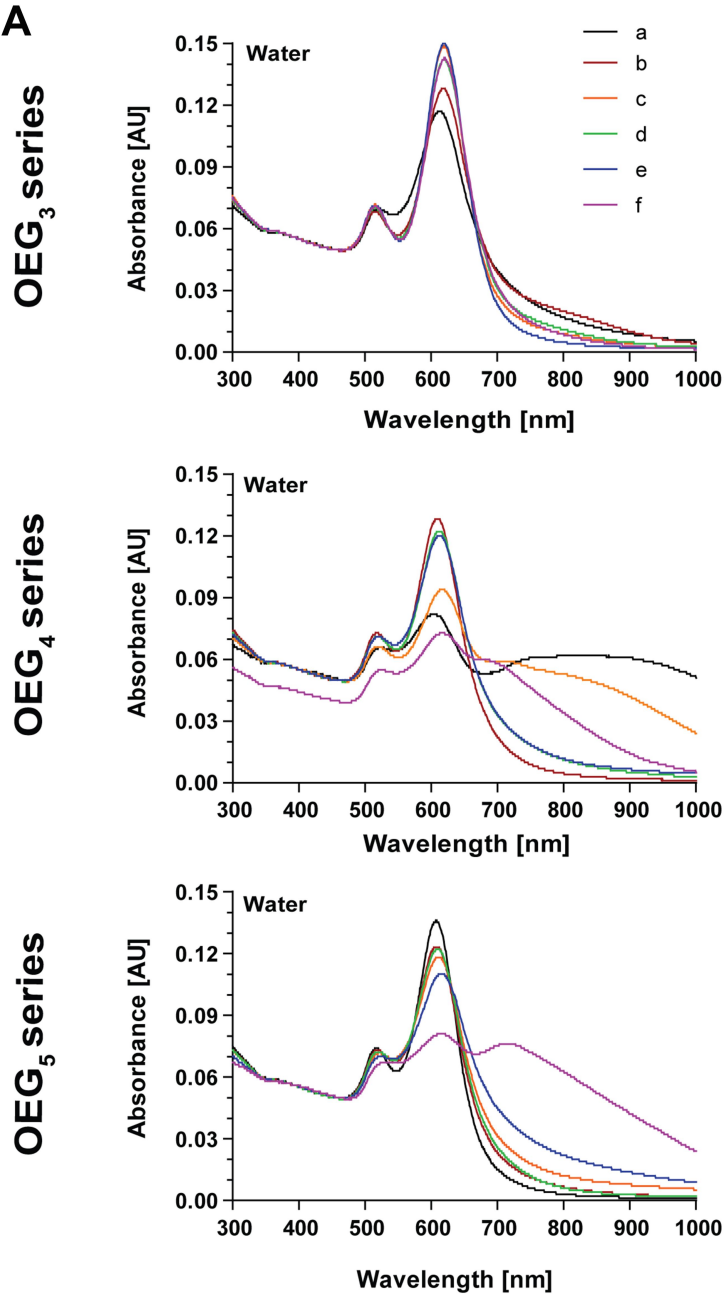

C
B
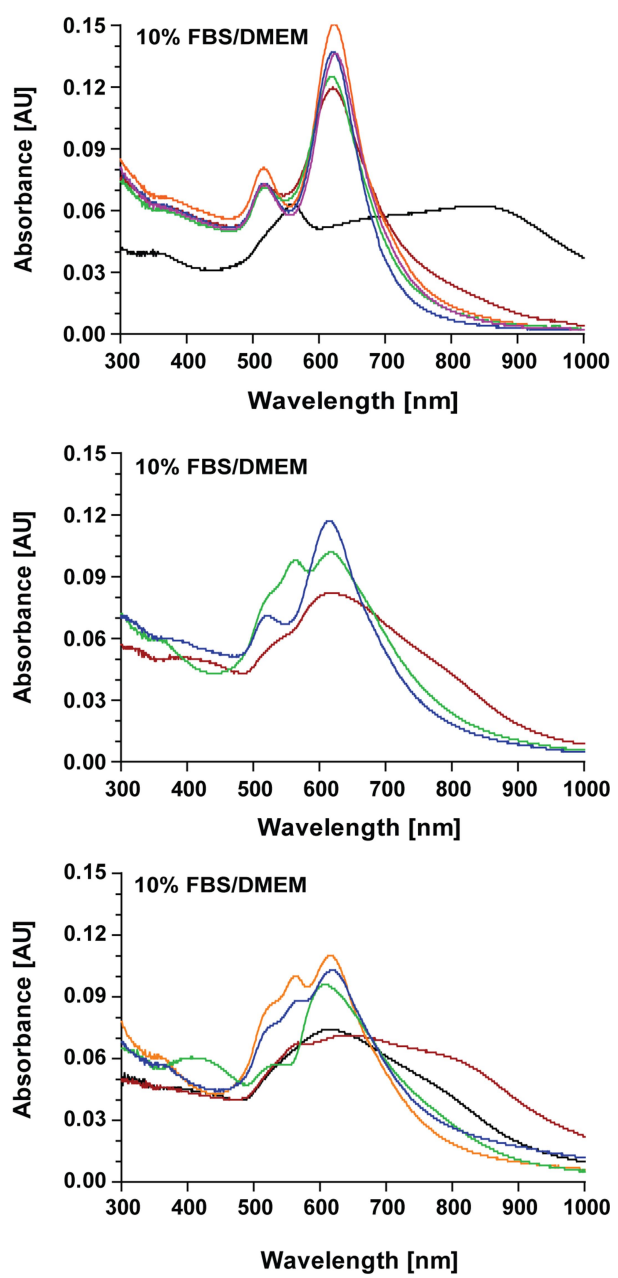

Wavelength [nm]

Alkyl ligand QAS group:

$\mathrm{OEG}_{3}$ series $\triangle \nabla \mathrm{a}$

$\mathrm{OEG}_{4}$ series एण b

OEG $_{5}$ series $\quad \cdots$ c

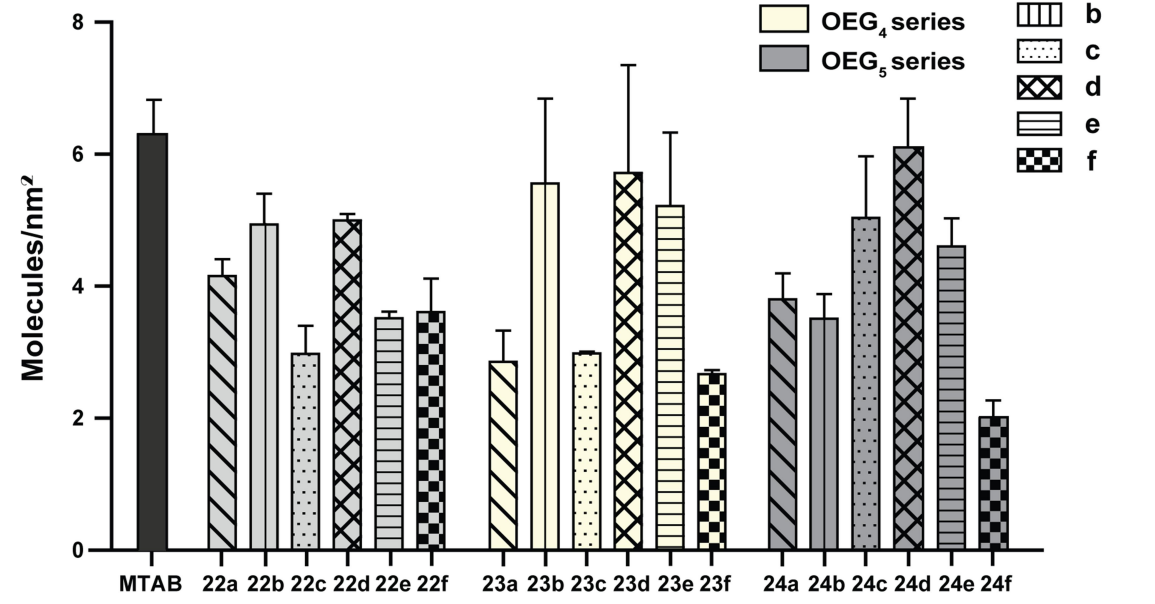

Ligands

Figure 3 Colloidal stability and ligand packing density of GNRs stabilized by cationic OEG compounds. (A) UV-VIS-NIR spectra of GNRs modified by OEG compounds with 3,4 , and 5 ethylene glycol units $\left(\mathrm{OEG}_{3}, \mathrm{OEG}_{4}\right.$, and $\mathrm{OEG}_{5}$ series) and various quaternary ammonium heads (a-f) after surface ligand exchange in storage solution (water) normalized to $50 \mu \mathrm{M}\left(\mathrm{Au}^{\circ}\right)$ concentration and (B) dispersed in $10 \% \mathrm{FBS} / \mathrm{DMEM}$ at $50 \mu \mathrm{M}\left(\mathrm{Au}^{\circ}\right)$ concentration. (C) Quantification of ligand packing density on GNRs using ICP-OES. 
aromatic compounds and the compound with aromatic side chain (b-f) led to slightly higher cytotoxicity (Supplementary Figure S2B), but still nearly two orders of magnitude better to MTAB. This finding leads to the conclusion that compounds with OEG chain are, in general, less toxic and more suitable for usage in living cells than the compounds with alkyl chain.

\section{Estimation of Cellular Uptake of ${ }^{\mathrm{OEG}+}$ GNRs}

The cellular uptake of GNRs modified by different ligands was evaluated in HeLa cells using confocal microscopy at a $20 \mu \mathrm{M}\left(\mathrm{Au}^{0}\right)$ GNRs concentration. The experiment confirmed that the ${ }^{\mathrm{OEG}+}$ GNRs are effectively absorbed by cells and localized into the perinuclear space (Figure 4A). Moreover, for all cationic GNRs, transport of GNRs into lysosomal compartment was confirmed by a co-association with lysosomal marker LAMP-1 (Supplementary Figure S7). Next, the GNRs level was semi-quantitatively estimated by fluorescence-activated cell sorting (FACS; Figure 4B). The median of scattered intensity of OEG ${ }^{+}$GNRs taken up by the cell was normalized to the signal of ${ }^{\mathrm{MTAB}} \mathrm{GNRs}$ known for their highly efficient cellular uptake. $^{47,48}$ In line with microscopic analyses, the FACS results showed a trend to increase the cellular absorption of ${ }^{\mathrm{OEG}+}$ GNRs depending on the length of the side chain of cationic ligand, where the ligands with four $\left(\mathrm{OEG}_{4}\right)$ and five $\left(\mathrm{OEG}_{5}\right)$ ethylene glycol units in the chain were taken up more effectively compared to ligands with three ethylene glycol units in the chain $\left(\mathrm{OEG}_{3}\right)$. In addition, the level of $\mathrm{OEG}_{4}$ and $\mathrm{OEG}_{5}$ series ligands reached or exceeded the intensity of alkyl chain ligand (MTAB). Nevertheless, any correlation of cellular uptake in dependency on the different cationic ligand head was not observed.

\section{Estimation of the Photothermal Stability of Differently Coated Cationic GNRs}

To assess the effect of the ligand structure on the photothermal properties of GNRs, we used synthesized GNRs tuned to near-infrared (NIR) region $(81.5 \pm 24.2 \mathrm{~nm}$ in length and 27.9 $\pm 3.4 \mathrm{~nm}$ in width; see Supplementary Figure S4B and $\underline{\mathrm{D}}$ for size distribution of GNRs and their UV-Vis-NIR spectra, respectively), where tissues exhibit lower absorption. ${ }^{4,69}$ As the results obtained by FACS analysis showed that the uptake of GNRs by cells increases with the length of OEG chain, the ligands from the series with the highest number of ethylene glycol units $\left(\mathrm{OEG}_{5}\right)$ were selected for subsequent evaluation of photothermal stability. From $\mathrm{OEG}_{5}$ series, ligands providing the highest surface coverage density and satisfactory colloidal stability of GNRs both in water and cell culture medium (24c and $\mathbf{2 4 d}$ ) were evaluated as the most promising and selected as the representative samples. 24c- and 24dcoated GNRs (see Supplementary Figure S5B for UV-VisNIR spectra of ligand-modified GNRs) were deposited on TEM grids and then were irradiated by 140 fs pulses with 80 $\mathrm{MHz}$ repetition rate, central wavelength of $750 \mathrm{~nm}$, and peak fluence set to $14.8 \mathrm{~mJ} / \mathrm{cm}^{2}, 45.0 \mathrm{~mJ} / \mathrm{cm}^{2}$, and $142.5 \mathrm{~mJ} / \mathrm{cm}^{2}$ on two-photon excitation microscope. The sets of samples were characterized prior to and after the laser irradiation on FE-SEM using specialized particle shape recognition software package $^{70}$ (see Supplementary Figures S8 and S9). Morphological stability of metal nanoparticles of ${ }^{24 \mathrm{c}} \mathrm{GNR}$ and ${ }^{24 \mathrm{~d}}$ GNRs was compared to ${ }^{\mathrm{MTAB}}$ GNRs. As revealed in Table 1, the highest fluence $\left(142.5 \mathrm{~mJ} / \mathrm{cm}^{2}\right)$ caused reshaping of all GNRs regardless of their solvation layer due to transient accumulation of heat in or around GNRs. In all cases, reshaping of GNRs resulted in a decrease of rod length and their aspect ratio (Figure 5A). However, for $\mathbf{2 4 d}$-coated GNRs, the fluence of $14.8 \mathrm{~mJ} / \mathrm{cm}^{2}$ triggered already morphological changes of GNRs as indicated by a decrease of GNRs aspect ratio from $2.7 \pm 0.5$ to $2.1 \pm 0.4$ and a decrease of GNRs length from $28.6 \pm 4.0$ to $23.8 \pm 4.2$. Similarly, the photothermal stability of 24c-coated GNRs saturated already at 45.0 $\mathrm{mJ} / \mathrm{cm}^{2}$ (see Figure 5B), when aspect ratio of GNRs decreased from $2.7 \pm 0.5$ to $1.6 \pm 0.5$ and rods length from $29.1 \pm 4.0$ to $20.8 \pm 4.4$. Under femtosecond laser irradiation, the reshaping of GNRs is competitive to the thermal diffusion across the shell layer of NPs. ${ }^{71}$ Our results thus indicate lower thermal stability and lower thermal transport into the surrounding environment from heated ${ }^{\mathrm{OEG}+}$ GNRs in comparison to alkanethiol-stabilized ${ }^{\mathrm{MTAB}}$ GNRs.

\section{Discussion}

\section{Rationale and Design of Quaternary Ammonium Salts with OEG Chain}

Due to high application potential of GNPs in clinical medicine, ${ }^{11,72-74}$ in this study we prepared various OEG compounds containing the positive charge in the terminal group. Our aim was to improve the cellular uptake of GNRs compared to PEGylated GNRs and to decrease the toxicity of free surfactant compared to alkanethiols. Moreover, it was hypothesized that the ligands with the OEG-chains will provide a less compact passivating layer due to their ability to absorb molecules of water, which could lead to conveniently modified photothermal 


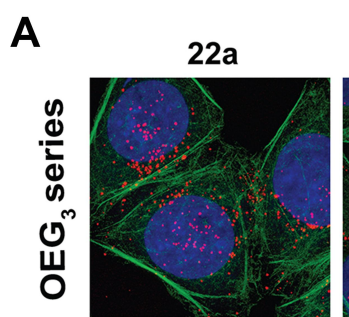

造

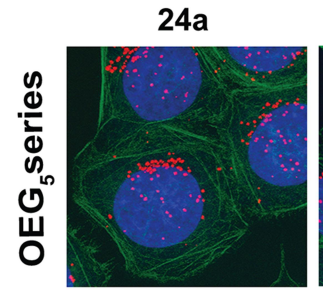

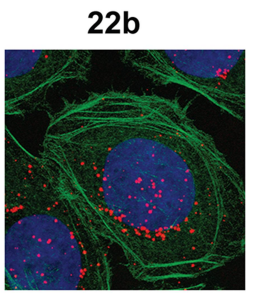

$23 b$

22c
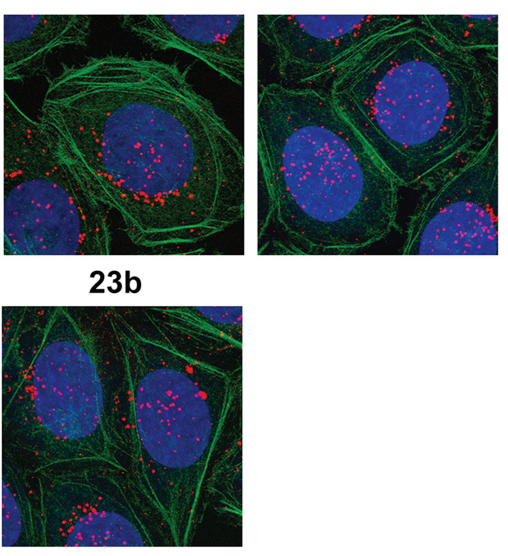

24b
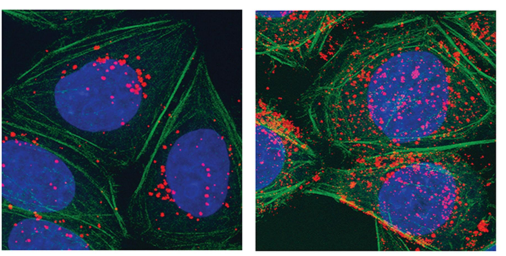

22d

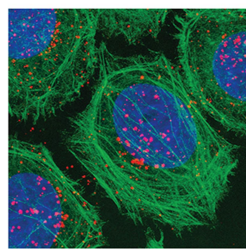

23d

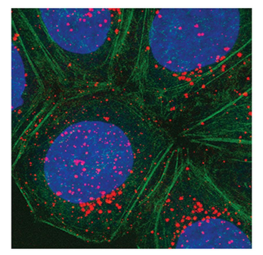

24d

$22 e$

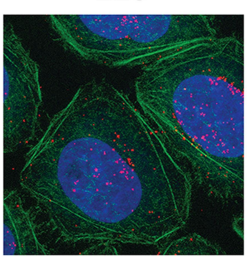

$23 e$

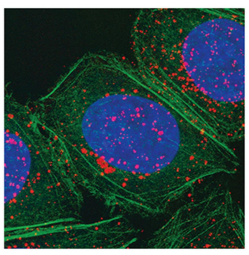

$24 e$
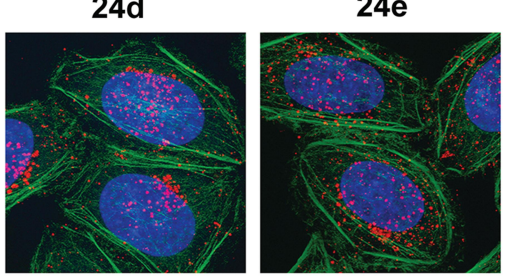

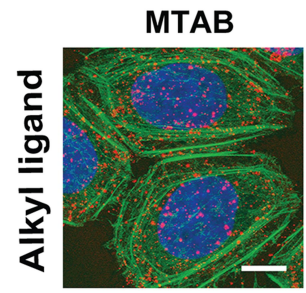

B
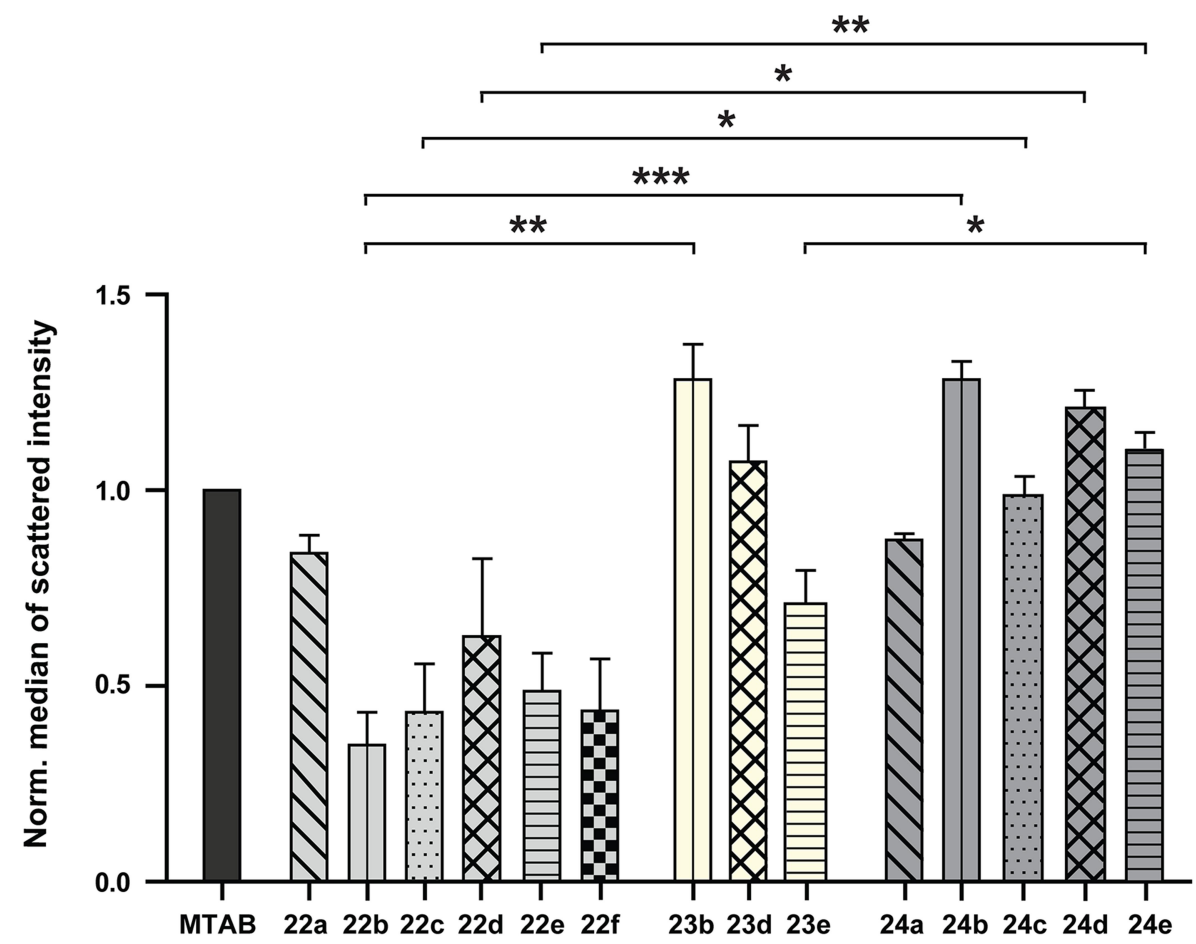

QAS group:

$\Delta$ a

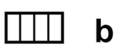

$\therefore$ c

Q d

E $e$

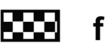

\section{Ligands}

Figure 4 Efficiency of cellular uptake and intracellular localization of GNRs stabilized by QAS. (A) Cellular uptake of various cationic GNRs modified by ligands with OEG $\left(\mathrm{OEG}_{3}, \mathrm{OEG}_{4}\right.$, and $\mathrm{OEG}_{5}$ series) chain and different terminal QAS group (a-f) and by QAS-ligand with alkyl chain (MTAB) determined by confocal microscopy in HeLa cells incubated with $20 \mu \mathrm{M}\left(\mathrm{Au}^{0}\right)$ GNRs for 24 hours (a back-scattered light from the longitudinal LSPR mode of GNRs was utilized to visualize GNRs; the actin and nuclei were stained by phalloidin and DAPI, respectively; whole cells were scanned as a series of z-stacks) and (B) semi-quantified by FACS in HeLa cells after 24 h-incubation with $20 \mu M$ $\left(\mathrm{Au}^{0}\right) \mathrm{GNRs}$ (the median of scattered intensity of ${ }^{\mathrm{OEG}+}$ GNRs was normalized to ${ }^{\mathrm{MTAB}} \mathrm{GNRs}$ ). Bar, $10 \mu$ m; Student's $t$-test: $P<0.05$ ( $\left.{ }^{*}\right), P<0.0 \mathrm{I}(* *)$, and $P<0.00 \mathrm{I}(* * *)$. 


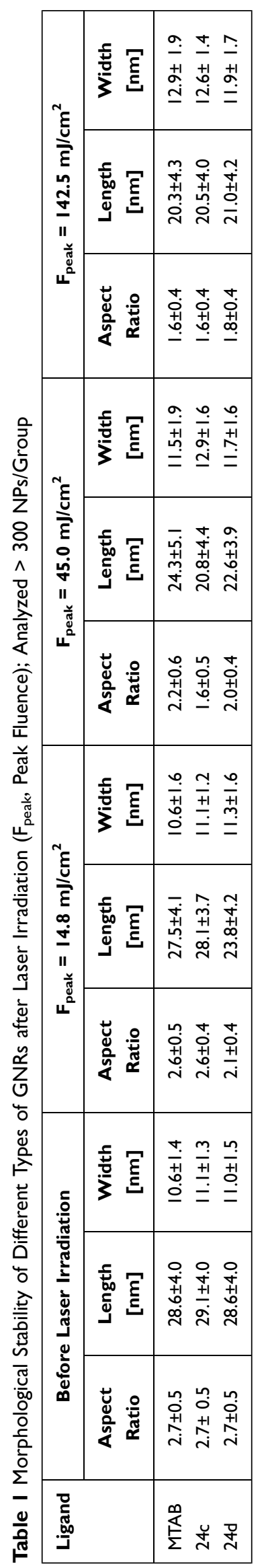

characteristics. ${ }^{15,75,76}$ Vigderman et $\mathrm{al}^{47}$ previously demonstrated that cationic alkanethiol ligand MTAB of the same length of alkane chain as a CTAB used to cover the GNRs during synthesis allows the complete ligand exchange and highly increases the colloidal stability of GNRs. Therefore, here we designed the analogues of MTAB (C16) with OEG chain considering the divergent bond lengths, bond angles, and spatial arrangement of atoms in molecules. The OEG/PEG can adopt helical or all-trans conformation and ratio between these two forms is a function of many factors. The attachment of (EG)n via sulfur group on flat gold surface allows both conformation such as for $n \geq 6$ helical and for $n=4$ a mix of helical and all-trans conformations. ${ }^{77,78}$ Based on that, OEG compounds with three different lengths of chain (22-24) and with six various quaternary ammonium heads were prepared: QAS with sole aliphatic substitution (quaternary heads a, d) or as a part of heteroaromatic system (quaternary heads $\mathbf{b}, \mathbf{c}, \mathbf{e}, \mathbf{f})$. The various properties of QAS such as the different conformation of OEG chain, ESP charge distribution and hydrophobicity could have an important influence on the ligand cytotoxicity and its packing density on GNRs and thus on the GNRs colloidal stability, cellular uptake, as well as on the photothermal properties of GNRs.

\section{Cytotoxicity of Cationic OEG Ligands}

The utilization of thiol-containing compounds as the ligand for GNRs stabilization may also exhibit some disadvantages. Thiol-containing small molecules such as dithiothreitol or amino acids such as methionine and cysteine at physiological concentrations can cause release of the thiol ligand and subsequently bind to vacant adsorption sites on the nanoparticle surface. ${ }^{15,79,80}$ In contrast to the formerly accepted view, recent research demonstrating that the gold-sulfur coupling in gold-thiol self-assembled monolayers (SAMs) has a physisorbed rather than chemisorbed (or covalent) character supports the possibility of ligand release. ${ }^{81,82}$ Moreover, a ligand desorption can be caused by heating ${ }^{83}$ and by an intensive fs-laser irradiation of GNPs, leading to their reshaping and melting that trigger the breaking of the S-Au bond. ${ }^{84,85}$ In the blood stream, the released free ligands can then cause toxicity or induce tissue pathology. ${ }^{86-89}$ Despite the GNRs being capped with cationic alkanethiol (MTAB) $)^{47,66}$ as well as OEG ligands being evaluated non-cytotoxic, the excessive ligand release during the exalted or repeated GNRs dose or photothermal therapy may increase the probability of free 
A
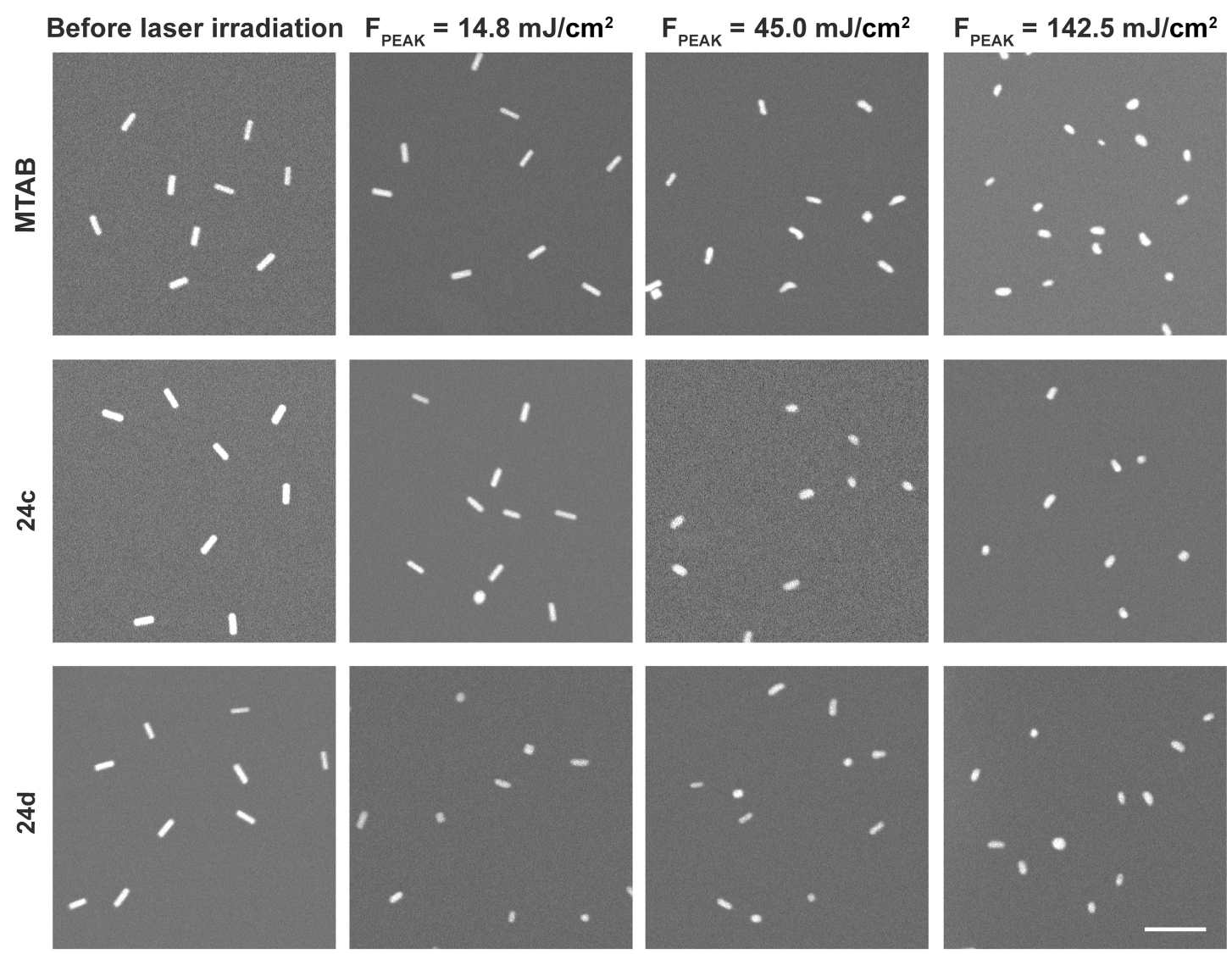

B

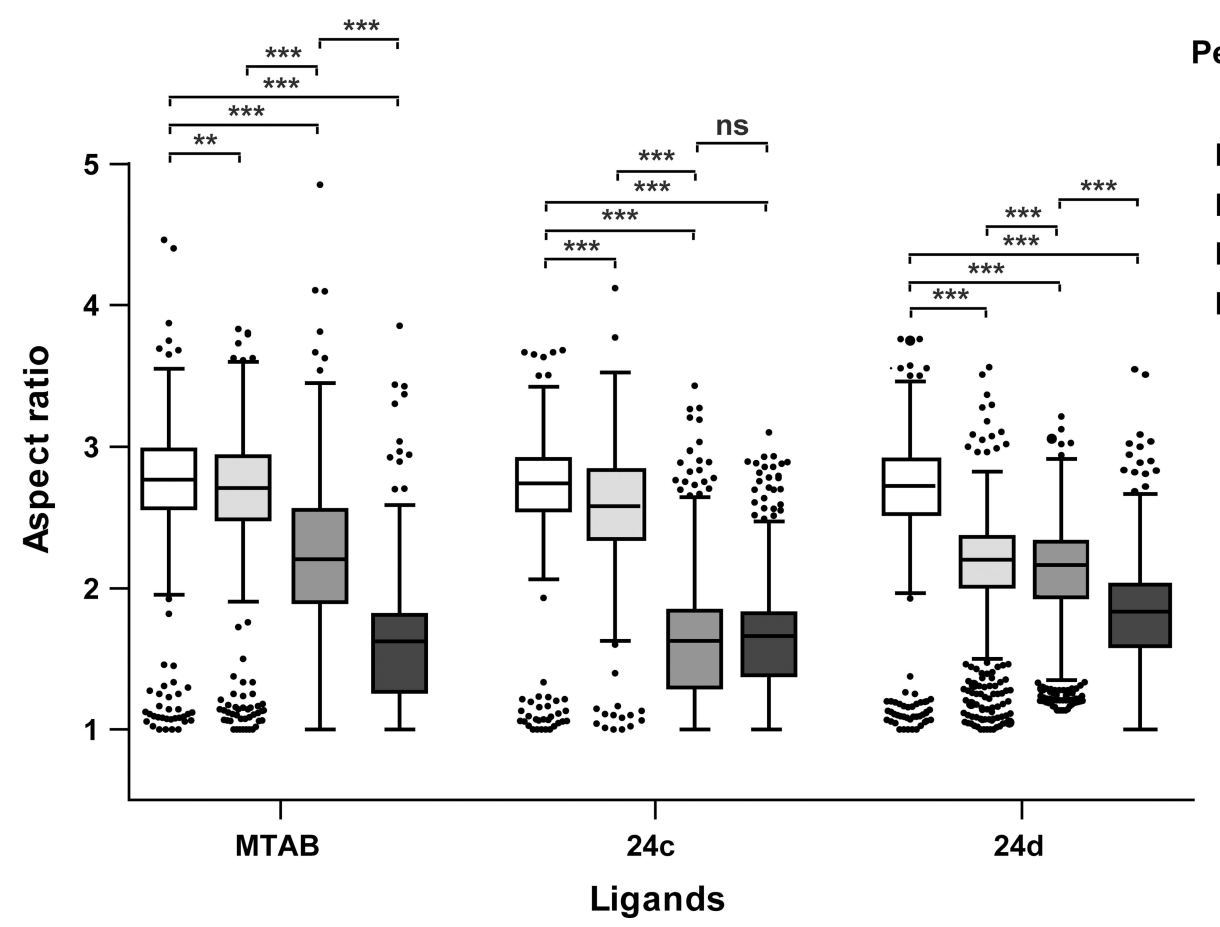

Peak fluence

$\left[\mathrm{mJ} / \mathrm{cm}^{2}\right]$

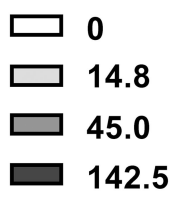

Figure 5 Photothermal stability of GNRs coated by selected ligands. (A) Representative FE-SEM images of MTAB, 24c-and 24d-coated GNRs (tuned to NIR region) before
and after two-photon irradiation by $140 \mathrm{fs}$ pulses with $80 \mathrm{MHz}$ repetition rate at wavelength of $750 \mathrm{~nm}$ and peak fluence $\left(\mathrm{F}_{\mathrm{PEAK}}\right)$ set to I4.8 mJ/cm², $45.0 \mathrm{~mJ} / \mathrm{cm}^{2}$, and I42.5 $\mathrm{mJ} / \mathrm{cm}^{2}$ (bar, $250 \mathrm{~nm}$ ). (B) Tukey box plot showing median of aspect ratio of GNRs (solid line inside the box) before and after the laser irradiation for MTAB, 24c and 24d and three values of laser peak fluence. Whiskers extend to outliers up to 1.5-times the interquartile range and further outliers are marked as dots above boxes; Student's $t$ test: not significant (ns), $P<0.01$ (**), and $P<0.001$ (***). 
ligand toxicity in vivo. For this reason, the cell viability assay of free compounds was performed on non-cancerous CHO-K1 cells. In general, the cytotoxicity of quaternary ammonium compounds increases with their increasing hydrophobicity, mainly attributable to their efficient incorporation into cell phospholipid membrane and formation of mixed micelles with membrane constituents causing the membrane ruptures and leakage of cytosol. ${ }^{49,90,91}$ Our results showed that all synthesized QAS with OEG chain exhibit almost two orders of magnitude lower cytotoxicity compared to conventional QAS with the alkyl chain, which is in agreement with lower hydrophobicity of OEG compounds. Similarly, OEG compounds containing aliphatic trimethylammonium group that showed the lowest hydrophobicity of the QAS heads also exhibited nearly one order of magnitude lower toxicity against cells when combined with the chain of four and five ethylene glycol units $\left(\mathrm{OEG}_{4}\right.$ and $\left.\mathrm{OEG}_{5}\right)$. The introduction of aromatic system led to a slight increase of cytotoxicity when the cytotoxic effect was endorsed by the prolongation of OEG chain, which is also in agreement with the hydrophobic descriptors (Supplementary Table S1 and Supplementary $\underline{\text { Figure S2B). }}$.

\section{Colloidal Stability Studies of GNRs Modified by Cationic OEG Compounds}

Agglomeration/aggregation may dramatically affect nanoparticle properties which can mediate their biological effects. ${ }^{92,93}$ This underlines the importance of evaluation of colloidal stability in relevant media such as water or cell culture medium. Although most prepared OEG ligands provided stable colloidal dispersion of GNRs in the water, ${ }^{\mathrm{OEG}+} \mathrm{GNRs}$ tended to aggregate in the serum-containing cell culture medium. It is known that the blood serum, a cocktail of proteins, is amenable to unguided attachment onto NPs and is responsible for the colloidal stability or anti-aggregation effect. ${ }^{48,94}$ Thus the ability of OEG chain to reduce protein adsorption on the surface of $\mathrm{NPs}^{14}$ induces likely lower GNRs colloidal stability in the cell culture medium where the high content of salts promotes their aggregation. ${ }^{95}$

In addition, the ligand packing density on NPs plays an important role. ${ }^{96,97}$ In agreement with a previous report showing that the grafting densities of high molecular weight PEG on GNP are significantly lower than those of thiols ${ }^{80}$ we observed lower GNRs coverage also by most of OEG compared to ligands with alkyl chain of similar length. Lower number of cationic nitrogen groups bound to the surface of GNRs that provides the electrostatic repulsion between NPs may result in lower colloidal stability of GNRs in aqueous environment. ${ }^{18}$ In relation to the different structure of cationic head, the benzalkonium salts (22-24d) that provided good colloidal stability of GNRs in the cell culture medium also showed the highest packing density on GNRs. Surprisingly, the different length of OEG chain did not show any significant differences in ligand coverage of GNRs, while the colloidal stability of GNRs decreased with the number of ethylene glycol units. Likely the structural diversity of OEG compounds itself may lead to different orderliness of organic shell on GNRs surface due to different intermolecular forces and thus to diverse tendency to form aggregates in aqueous environment. The differing structure of quaternary ammonium head led to different inter- and intra-molecular interaction, such as electron-donating/ withdrawing effects of substituents, aromatic $\pi-\pi$ interactions or cation- $\pi$ interactions. ${ }^{97-99}$ The bulky positively charged head groups can control ligand density via volume of the terminal groups and repulsive forces between them. ${ }^{47,97,100}$ For example, ligand without aromatic moiety 24a exhibited almost half coverage of analogous ligand with aromatic ring 24d despite both substituents having similar $\mathrm{N}_{\mathrm{ESP}}$ and thus should be presumed to have similar repulsion of cationic parts. Moreover, the ligand 24d can occupy larger space due to the presence of aromatic ring. The introduction of aromatic ring itself can therefore contribute to colloidal stabilization of the ligand packedGNRs and the flexible linkers can help to avoid a jam of head groups by slight alteration of their conformations. ${ }^{100}$

\section{Cellular Uptake and Intracellular Localization of Cationic OEG-Modified GNR}

The cellular uptake of GNRs was firstly studied by confocal microscopy including the determination of GNRs localization and subsequently quantified by FACS. Similarly to ${ }^{\mathrm{MTAB}} \mathrm{GNRs},{ }^{48} \mathrm{OEG}^{+}$GNRs adhered onto the cell plasma membrane were engulfed and trafficked to lysosomes. A semi-quantitative estimation revealed that the cellular uptake of ${ }^{\mathrm{OEG}+}$ GNRs increases with the length of OEG chain and thus with increasing hydrophobicity of the ligand. This is consistent with a study by Lorenz et al, ${ }^{101}$ demonstrating that with the increasing hydrophobicity of a linear alkyl side chain the absorption 
of poly(alkyl methacrylate) nanoparticles by HeLa, primary human bone marrow-derived MSCs, KG1a, and Jurkat cells significantly increases. However, no correlation was observed in relation to differences in cationic heads that also provide a different degree of hydrophobicity. It has been shown that cationic surface charge enhances the cellular absorption of NPs compared to anionic and neutral charge. ${ }^{24,94}$ However, the initial surface charge may not be a simple predictor of nanoparticle uptake. Against expectation, the methyl-containing compounds that exhibited the highest EPS partial charge on the quaternary nitrogen did not show the highest level of cellular absorption in all tested series. On the other hand, the cellular uptake of ${ }^{\mathrm{OEG}+}$ GNRs correlated with decreasing stability of GNRs in the cell culture medium and with increasing formation of nanoparticle aggregates. Previously, we showed that the aggregated nanorods are endocytosed via macropinocytosis, while clathrinmediated endocytosis (CME) is a preferential mechanism for individual nanorods. ${ }^{48}$ Therefore, the enhanced tendency to form aggregates may increase the amount of internalized GNRs on behalf of macropinocytosis, that is the dominant endocytic pathway for NPs. ${ }^{48,102}$ Thus, the affinity to form aggregates was probably a major factor determining the mechanism and level of cellular uptake of ${ }^{\mathrm{OEG}+}$ GNRs.

\section{Thermal Stability and the Heat Transport from GNRs during Exposure to Femtosecond Laser Irradiation}

The thermal therapy by GNPs promises progress in noninvasive cancer treatment. ${ }^{103,104}$ Upon interaction of GNPs with the laser pulses, the rate of heat dissipation from the hot metal particle to the surrounding media determines the efficiency of the thermal killing of cancer cells. In case of metal NPs coated with water soluble surfactant, the effective thermal conductance $\left(\mathrm{G}_{\text {eff }}\right)$ includes the thermal conductance of the metal/surfactant interface, the intrinsic thermal conductance of the surface molecules, and the thermal conductance of the surfactant/water interface. ${ }^{44}$ Previously, the transient absorption measurements as well as computer simulations were applied to study the heat transfer from GNPs to their surroundings. ${ }^{39,40,43,44}$ Here, we utilized the method evaluating the photothermal stability of GNRs under the femtosecond laser irradiation. Particle cooling depends on the metal/surfactant interface thermal conductance. The excessive accumulation of heat energy may result in NPs fragmentation or changes in their size and shape. ${ }^{30,34,71}$ This approach allows to determine a risk of secondary cytotoxic and genotoxic effects of "reshaped" NPs on cells surviving the laser irradiation and neighbouring/unexposed cells.

According to the surface ligand properties, several studies reported higher thermal conductivity for ligands with higher hydrophilicity that brings associated water molecules near the GNRs surface. ${ }^{40,43,44}$ As the direct contact between water molecules and gold provides an additional thermal path, the thermal conductance of the Au/hydrophilic PEG ( 23 ethylene glycol units) interface comprises $\mathrm{G}_{\text {Au-PEG }}$ and $\mathrm{G}_{\mathrm{Au} \text {-Water }}$ components. While the calculated value of $\mathrm{G}_{\mathrm{Au}-\mathrm{PEG}}$ is comparable to the $\mathrm{Au} /$ lipophilic alkanedithiol interfacial thermal conductance, ${ }^{105,106}$ the additional thermal path between $\mathrm{Au}$ and water $\left(\mathrm{G}_{\mathrm{Au} \text {-Water }}\right)$ increased the overall thermal conductivity of the GNR-PEG system about half. ${ }^{44}$ Besides the hydrophilicity, the type of solvent, ${ }^{107}$ bond strength between the $\mathrm{Au}$ and surface ligand ${ }^{44}$ as well as ligand packing density ${ }^{40,108}$ determine the efficiency of the heat transport from the GNPs. In case of CTABstabilized GNRs, the thermal interface conductance decreased with the increasing concentration of free hydrophobic CTAB. ${ }^{108}$ In contrast, for hydrophilic molecules, the thermal conductivity of the GNRs polyelectrolyte layer increased with the increasing number of poly (acrylic acid) (PAA) layers and slightly decreased with increasing number of polyallylamine hydrochloride (PAH) layers. ${ }^{40}$ Our results showed that despite less hydrophobic OEG-chain, the cationic OEG-GNRs lost the photothermal stability at lower laser fluence and thus heat transport to the surrounding was less effective than for similar cationic alkanethiol-GNRs. The difference in ligand packing density could lead to a discrepancy in the rate of heat dissipation. However, the OEG ligands used for evaluation of photothermal stability reached nearly analogous ligand packing density as MTAB. This indicates that also other factors that reflect the divergent chemical structures of MTAB and OEG compounds such as different assembly kinetics of thiolated ligands and orderliness of the formed ligand shell may influence the photothermal properties of GNRs. ${ }^{109}$

In terms of thermal effectiveness, the OEG-GNRs are thus suitable for photothermal therapy to induce cancer cell death by programmed cell death pathway - 
apoptosis, with avoidance of necrosis. ${ }^{104}$ Upon these settings, the cells are heated only to a temperature around $42-43^{\circ} \mathrm{C} .{ }^{110}$ However, lower heat dissipation and higher risk of NPs fragmentation in applications requiring irradiation by high laser intensity such as tissue micro-surgery ${ }^{111,112}$ may lead to lower effectiveness and cytotoxic and genotoxic damage of nanoparticle remnants.

\section{Conclusion}

In conclusion, the physico-chemical and biological properties of 18 newly-designed and synthesized cationic OEG compounds have been examined as ligand shells of GNRs and compared to each other as well as to their alkyl analogue - MTAB (summarized in Table 2). Our results showed that while the ability of cationic OEG ligands to stabilize the colloidal dispersion of GNRs in water and in serum-containing cell culture medium decreases with length of OEG chain, the cellular uptake of GNRs by cancer cells increases. The most promising QAS group appears to be the benzalkonium moiety that allowed to prepare GNRs with sufficient colloidal stability, high ligand packing density and reasonable cellular uptake in combination with all tested chain lengths. In comparison to the alkyl-QAS ligand, all OEG compounds exhibited nearly two orders of magnitude lower cytotoxicity in free state. Despite of the aggregation in cell culture medium, the series of OEG ligands with 4 and 5 ethylene glycol units in the chain revealed the comparable level of GNRs absorbed by cell as their alkyl analogue MTAB. The effect of ligand shell on the heat transport from GNRs under fs-laser irradiation was determined using a novel approach quantifying the reshaping of more than 300 GNRs by software analysis of FE-SEM micrographs that allows the assessment of biological risk of fragmented GNRs. However, the thermal stability of ${ }^{\mathrm{OEG}+}$ GNRs and thus the heat dissipation to the surrounding media was lower than for ${ }^{\mathrm{MTAB}}$ GNRs. Based on these divergent properties, ${ }^{\mathrm{OEG}}$ ${ }^{+}$GNRs appear to be optimal for clinical applications requiring systemic administration of NPs/NPs treated cells such as drug delivery and photothermal therapy inducing apoptosis, while the alkanethiol-modified GNRs may be more suitable for local application with high intensity laser irradiation such as tissue microsurgery.

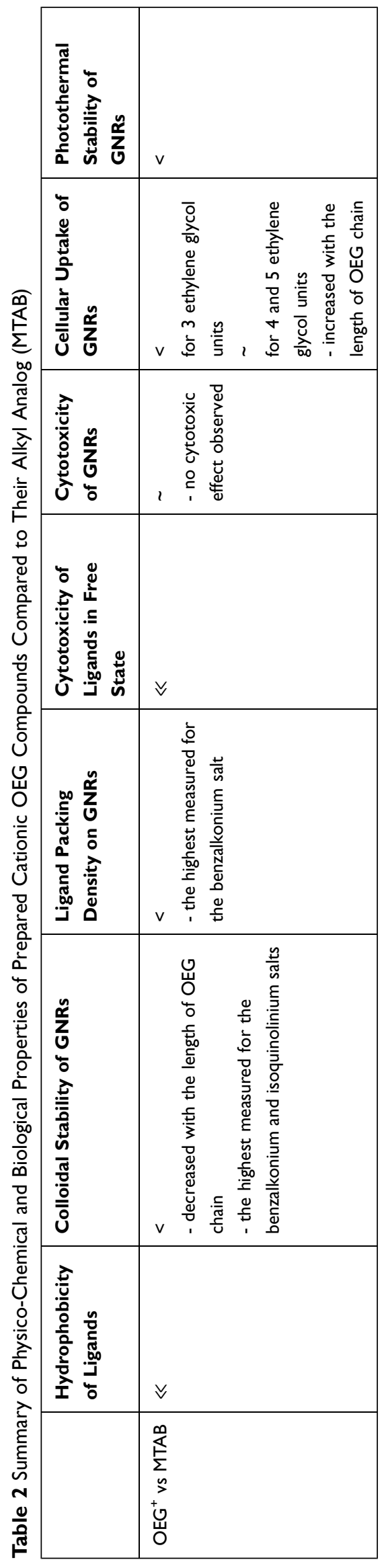




\section{Abbreviations}

$\mathrm{AcCl}$, acetyl chloride; $\mathrm{ACN}$, acetonitrile; cpd, compound; CTAB, cetyltrimethylammonium bromide; DCE, 1,2-dichloroethane; DCM, dichloromethane; DCM, dichloromethane; DMAP, 4-(dimethylamino)pyridine; DMEM, Dulbecco's Modified Eagle's Medium; DMTrCl, 4,4'dimethoxytrityl chloride; EDTA, ethylenediaminetetraacetic acid; ESP, molecular electrostatic potential; EtOAc, ethyl acetate; FACS, fluorescence-activated cell sorting; FBS, fetal bovine serum; FE-SEM, field emission scanning electron microscopy; G, thermal conductance; GNPs, gold nanoparticles; GNRs, gold nanorods; $\mathrm{IC}_{50}$, half maximal inhibitory concentration; ICP-OES, inductively coupled plasma - optical emission spectrometry; KSAc, potassium thioacetate; LAMP-1, lysosomal associated membrane protein 1; LC-MS, liquid chromatography-mass spectrometry; LSRP, localized surface plasmon resonance; MEK, methyl ethyl ketone; $\mathrm{MeOH}$, methanol; MTT, 3-(4,5-dimethylthiazol-2-yl)-2,5-diphenyltetrazolium bromide; NIR, near-infrared region; NMR, nuclear magnetic resonance; OEG, oligoethylene glycol; PBS, phosphate buffered saline; PEG, polyethylene glycol; POSAB, $N, N, N$-trimethyl-3,6,9,12,15pentaoxaheptadecyl-17-sulfanyl-1-ammonium bromide; QAS, quaternary ammonium salts; $\mathrm{PPh}_{3}$, triphenylphosphine; $p$-TsCl, $p$-toluenesulfonyl chloride; r.t., room temperature; TCA, trichloroacetic acid; TEA, triethylamine.

\section{Data Sharing Statement}

The authors confirm that the data supporting the findings of this study are available within the article and its supplementary materials.

\section{Acknowledgment}

The work was supported by the Grant Agency of the Czech Republic (project no. GA16-13967S), Ministry of Health of the Czech Republic (grant nr. NV19-08-00523), Institutional Grant (project no. RVO 68378050), University of Hradec Kralove (Faculty of Science no. VT2019-2021), Czech Technical University in Prague (project no. RVO 68407700; project CAAS no. CZ.02.1.01/0.0/0.0/16_019/ 0000778) and by the project Advanced Functional Nanorobots (reg. no. CZ.02.1.01/0.0/0.0/15_003/0000444 financed by the EFRR). We also acknowledge the MH CZ - DRO (University Hospital Hradec Kralove, no. 00179906), the First Faculty of Medicine of the Charles University in Prague, the BioImaging Facility, Institute of Physiology, CAS (supported by the Czech-BioImaging -
LM2015062 funded by MEYS CR), for two-photon laser exposition and the Light Microscopy Core Facility, IMG ASCR, Prague, Czech Republic, supported by MEYS (LM2015062，CZ.02.1.01/0.0/0.0/16_013/0001775) and OPPK (CZ.2.16/3.1.00/21547), for their support with the confocal imaging presented herein. The authors are grateful for ICP-OES measurement to Stanislava Matejkova; Institute of Organic Chemistry and Biochemistry of the Czech Academy of Sciences, Prague, Czech Republic.

\section{Author Contributions}

All authors made substantial contributions to the conception and design, acquisition of data, or analysis and interpretation of data; took part in drafting the article or revising it critically for important intellectual content; agreed to submit to the current journal; gave final approval of the version to be published; and agree to be accountable for all aspects of the work.

\section{Disclosure}

The authors reported no conflicts of interest for this work.

\section{References}

1. Huang X, El-Sayed MA. Gold nanoparticles: optical properties and implementations in cancer diagnosis and photothermal therapy. $J$ Adv Res. 2010;1:13-28. doi:10.1016/j.jare.2010.02.002

2. Elahi N, Kamali M, Baghersad MH. Recent biomedical applications of gold nanoparticles: a review. Talanta. 2018;184:537-556. doi:10.1016/j.talanta.2018.02.088

3. Bodelon G, Costas C, Perez-Juste J, Pastoriza-Santos I, LizMarzan LM. Gold nanoparticles for regulation of cell function and behavior. Nano Today. 2017;13:40-60. doi:10.1016/j. nantod.2016.12.014

4. Tong L, Wei Q, Wei A, Cheng JX. Gold nanorods as contrast agents for biological imaging: optical properties, surface conjugation and photothermal effects. Photochem Photobiol. 2009;85:21-32. doi:10.1111/j.1751-1097.2008.00507.x

5. Cao J, Sun T, Grattan KTV. Gold nanorod-based localized surface plasmon resonance biosensors: a review. Sens Actuators B Chem. 2014;195:332-351. doi:10.1016/j.snb.2014.01.056

6. Murphy CJ, Gole AM, Hunyadi SE, et al. Chemical sensing and imaging with metallic nanorods. Chem Commun. 2008:544-557. doi:10.1039/B711069C

7. Baffou G, Quidant R. Thermo-plasmonics: using metallic nanostructures as nano-sources of heat. Laser Photon Rev. 2013;7:171-187. doi:10.1002/lpor.201200003

8. Huang X, Jain PK, El-Sayed IH, El-Sayed MA. Plasmonic photothermal therapy (PPTT) using gold nanoparticles. Lasers Med Sci. 2008;23:217-228. doi:10.1007/s10103-007-0470-x

9. Pissuwan D, Niidome T, Cortie MB. The forthcoming applications of gold nanoparticles in drug and gene delivery systems. $J$ Control Release. 2011;149:65-71. doi:10.1016/j.jconrel.20 09.12 .006

10. Boulais E, Lachaine R, Hatef A, Meunier M. Plasmonics for pulsed-laser cell nanosurgery: fundamentals and applications. J Photochem Photobiol C. 2013;17:26-49. doi:10.1016/j. jphotochemrev.2013.06.001 
11. Rastinehad AR, Anastos H, Wajswol E, et al. Gold nanoshell-localized photothermal ablation of prostate tumors in a clinical pilot device study. Proc Natl Acad Sci. 2019;116:18590-18596. doi:10.1073/pnas.1906929116

12. Karakoti AS, Das S, Thevuthasan S, Seal S. PEGylated inorganic nanoparticles. Angew Chem Int Ed Engl. 2011;50:1980-1994. doi:10.1002/anie.201002969

13. Jokerst JV, Lobovkina T, Zare RN, Gambhir SS. Nanoparticle PEGylation for imaging and therapy. Nanomedicine. 2011;6:715-728. doi:10.2217/nnm.11.19

14. Pelaz B, Del Pino P, Maffre P, et al. Surface functionalization of nanoparticles with polyethylene glycol: effects on protein adsorption and cellular uptake. ACS Nano. 2015;9:6996-7008. doi:10.1021/acsnano. 5 b01326

15. Larson TA, Joshi PP, Sokolov K. Preventing protein adsorption and macrophage uptake of gold nanoparticles via a hydrophobic shield. ACS Nano. 2012;6:9182-9190. doi:10.1021/nn3035155

16. Alcantar NA, Aydil ES, Israelachvili JN. Polyethylene glycol-coated biocompatible surfaces. J Biomed Mater Res. 2000;51:343-351. doi:10.1002/1097-4636(20000905)51:3<343:: AID-JBM7>3.0.CO;2-D

17. Bagley AF, Hill S, Rogers GS, Bhatia SN. Plasmonic photothermal heating of intraperitoneal tumors through the use of an implanted near-infrared source. ACS Nano. 2013;7:8089-8097. doi: $10.1021 / \mathrm{nn} 4033757$

18. Zhang X, Servos MR, Liu J. Ultrahigh nanoparticle stability against salt, $\mathrm{pH}$, and solvent with retained surface accessibility via depletion stabilization. $J$ Am Chem Soc. 2012;134:9910-9913. doi:10.1021/ja303787e

19. Tirosh O, Barenholz Y, Katzhendler J, Priev A. Hydration of polyethylene glycol-grafted liposomes. Biophys $J$. 1998;74:1371-1379. doi:10.1016/S0006-3495(98)77849-X

20. Branca $C$, Magazù S, Maisano G, Migliardo F, Migliardo $P$, Romeo G. Hydration study of PEG/water mixtures by quasi elastic light scattering, acoustic and rheological measurements. J Phys Chem B. 2002;106:10272-10276. doi:10.1021/jp014345v

21. Suk JS, Xu Q, Kim N, Hanes J, Ensign LM. PEGylation as a strategy for improving nanoparticle-based drug and gene delivery. Adv Drug Deliv Rev. 2016;99:28-51. doi:10.1016/j. addr.2015.09.012

22. Hamidi M, Azadi A, Rafiei P. Pharmacokinetic Consequences of Pegylation. Drug Deliv. 2006;13:399-409. doi:10.1080/ 10717540600814402

23. Cho EC, Xie J, Wurm PA, Xia Y. Understanding the role of surface charges in cellular adsorption versus internalization by selectively removing gold nanoparticles on the cell surface with a I2/KI etchant. Nano Lett. 2009;9:1080-1084. doi:10.1021/n1803487r

24. Arvizo RR, Miranda OR, Thompson MA, et al. Effect of nanoparticle surface charge at the plasma membrane and beyond. Nano Lett. 2010;10:2543-2548. doi:10.1021/n1101140t

25. Zhang Y, Pan H, Zhang P, et al. Functionalized quantum dots induce proinflammatory responses in vitro: the role of terminal functional group-associated endocytic pathways. Nanoscale. 2013;5:5919-5929. doi:10.1039/c3nr01653f

26. Arnida J-AMM, Janát-Amsbury MM, Ray A, Peterson CM, Ghandehari H. Geometry and surface characteristics of gold nanoparticles influence their biodistribution and uptake by macrophages. Eur J Pharm Biopharm. 2011;77:417-423. doi:10.1016/j.ejpb.2010.11.010

27. Zhang YN, Poon W, Tavares AJ, McGilvray ID, Chan WCW. Nanoparticle-liver interactions: cellular uptake and hepatobiliary elimination. J Control Release. 2016;240:332-348. doi:10.1016/j. jconrel.2016.01.020

28. Sandhu KK, McIntosh CM, Simard JM, Smith SW, Rotello VM. Gold nanoparticle-mediated transfection of mammalian cells. Bioconjug Chem. 2002;13:3-6. doi:10.1021/bc015545c
29. Niidome T, Nakashima K, Takahashi H, Niidome Y. Preparation of primary amine-modified gold nanoparticles and their transfection ability into cultivated cells. Chem Commun. 2004;1978-1979. doi:10.1039/b406189f

30. Gao Z, Zhang L, Hu J, Sun Y. Mesenchymal stem cells: a potential targeted-delivery vehicle for anti-cancer drug, loaded nanoparticles. Nanomedicine. 2013;9:174-184. doi:10.1016/j. nano.2012.06.003

31. Kang S, Bhang SH, Hwang S, et al. Mesenchymal stem cells aggregate and deliver gold nanoparticles to tumors for photothermal therapy. ACS Nano. 2015;9:9678-9690. doi:10.1021/acsnano.5b02207

32. Mooney R, Roma L, Zhao D, et al. Neural stem cell-mediated intratumoral delivery of gold nanorods improves photothermal therapy. ACS Nano. 2014;8:12450-12460. doi:10.1021/nn505147w

33. Link S, Burda C, Mohamed MB, Nikoobakht B, El-Sayed MA. Laser photothermal melting and fragmentation of gold nanorods: energy and laser pulse-width dependence. $J$ Phys Chem A. 1999;103:1165-1170. doi:10.1021/jp983141k

34. González-Rubio G, Guerrero-Martínez A, Liz-Marzán LM. Reshaping, fragmentation, and assembly of gold nanoparticles assisted by pulse lasers. Acc Chem Res. 2016;49:678-686. doi:10.1021/acs.accounts.6b00041

35. Lapotko D. Plasmonic nanoparticle-generated photothermal bubbles and their biomedical applications. Nanomedicine. 2009;4:813-845. doi:10.2217/nnm.09.59

36. Lukianova-Hleb EY, Hanna EY, Hafner JH, Lapotko DO. Tunable plasmonic nanobubbles for cell theranostics. Nanotechnology. 2010;21:85102. doi:10.1088/0957-4484/21/8/085102

37. Letfullin RR, Joenathan C, George TF, Zharov VP. Laser-induced explosion of gold nanoparticles: potential role for nanophotothermolysis of cancer. Nanomedicine. 2006;1:473-480. doi:10.2217/ 17435889.1.4.473

38. Hartland GV. Optical studies of dynamics in noble metal nanostructures. Chem Rev. 2011;111:3858-3887.

39. Stocker KM, Gezelter JD. Simulations of heat conduction at thiolate-capped gold surfaces: the role of chain length and solvent penetration. $J$ Phys Chem C. 2013;117(15):7605-7612. doi: $10.1021 /$ jp312734f

40. Huang J, Park J, Wang W, Murphy CJ, Cahill DG. Ultrafast thermal analysis of surface functionalized gold nanorods in aqueous solution. ACS Nano. 2013;7:589-597. doi:10.1021/nn304738u

41. Soussi J, Volz S, Palpant B, Chalopin Y. A detailed microscopic study of the heat transfer at a water gold interface coated with a polymer. Appl Phys Lett. 2015;106:093113. doi:10.1063/1.4913905

42. Horiguchi Y, Honda K, Kato Y, Nakashima N, Niidome Y. Photothermal reshaping of gold nanorods depends on the passivating layers of the nanorod surfaces. Langmuir. 2008;24:12026-12031. doi:10.1021/la800811j

43. Alper J, Hamad-Schifferli K. Effect of ligands on thermal dissipation from gold nanorods. Langmuir. 2010;26:3786-3789. doi:10.1021/la904855s

44. Wu X, Ni Y, Zhu J, et al. Thermal transport across surfactant layers on gold nanorods in aqueous solution. ACS Appl Mater Interfaces. 2016;8:10581-10589. doi:10.1021/acsami.5b12163

45. Centi S, Cavigli L, Borri C, et al. Small thiols stabilize the shape of gold nanorods. J Phys Chem C. 2020;124:11132-11140. doi:10.1021/acs.jpcc.0c00737

46. Salajkova S, Sramek M, Malinak D, et al. Highly hydrophilic cationic gold nanorods stabilized by novel quaternary ammonium surfactant with negligible cytotoxicity. J Biophoton. 2019;12: e201900024. doi:10.1002/jbio.201900024

47. Vigderman L, Manna P, Zubarev ER. Quantitative replacement of cetyl trimethylammonium bromide by cationic thiol ligands on the surface of gold nanorods and their extremely large uptake by cancer cells. Angew Chem Int Ed Engl. 2012;51:636-641. doi:10.1002/anie.201107304 
48. Zarska M, Novotny F, Havel F, et al. A two-step mechanism of cellular uptake of cationic gold nanoparticles modified by (16-mercaptohexadecyl)trimethylammonium bromide (MTAB). Bioconjug Chem. 2016;27:2558-2574. doi:10.1021/acs. bioconjchem.6b00491

49. Benkova M, Soukup O, Prchal L, et al. Synthesis, antimicrobial effect and lipophilicity-activity dependence of three series of dichained N-alkylammonium salts. ChemistrySelect. 2019;4:12076-12084. doi:10.1002/slct.201902357

50. Nikoobakht B, El-Sayed MA. Preparation and growth mechanism of gold nanorods (NRs) using seed-mediated growth method. Chem Mater. 2003;15:1957-1962. doi:10.1021/cm0207321

51. Busbee BD, Obare SO, Murphy CJ. An improved synthesis of high-aspect-ratio gold nanorods. Adv Mater. 2003;15:414-416. doi:10.1002/adma.200390095

52. Edgar JA, McDonagh AM, Cortie MB. Formation of gold nanorods by a stochastic "popcorn" mechanism. ACS Nano. 2012;6:1116-1125. doi:10.1021/nn203586j

53. Elzey S, Tsai DH, Rabb SA, Yu LL, Winchester MR, Hackley VA. Quantification of ligand packing density on gold nanoparticles using ICP-OES. Anal Bioanal Chem. 2012;403:145-149. doi:10.1007/s00216-012-5830-0

54. Hinterwirth H, Kappel S, Waitz T, Prohaska T, Lindner W, Lammerhofer M. Quantifying thiol ligand density of self-assembled monolayers on gold nanoparticles by inductively coupled plasma-mass spectrometry. ACS Nano. 2013;7:11 29-1136. doi:10.1021/nn306024a

55. Verma A, Stellacci F. Effect of surface properties on nanoparticle-cell interactions. Small. 2010;6:12-21. doi:10.1002/ smll.200901158

56. Chompoosor A, Saha K, Ghosh PS, et al. The role of surface functionality on acute cytotoxicity, ROS generation and DNA damage by cationic gold nanoparticles. Small. 2010;6:22 46-2249. doi:10.1002/smll.201000463

57. Zhang J, Mou L, Jiang X. Surface chemistry of gold nanoparticles for health-related applications. Chem Sci. 2020;11:923-936. doi:10.1039/C9SC06497D

58. Liu X, Testa B, Fahr A. Lipophilicity and its relationship with passive drug permeation. Pharm Res. 2011;28:962-977. doi:10.1007/s11095-010-0303-7

59. Ungell AL, Nylander S, Bergstrand S, Sjoberg A, Lennernas H. Membrane transport of drugs in different regions of the intestinal tract of the rat. J Pharm Sci. 1998;87:360-366. doi:10.1021/ js $970218 \mathrm{~s}$

60. Goodwin JT, Conradi RA, Ho NF, Burton PS. Physicochemical determinants of passive membrane permeability: role of solute hydrogen-bonding potential and volume. $J$ Med Chem. 2001;44:3721-3729. doi:10.1021/jm010253i

61. Soukup O, Dolezal R, Malinak D, et al. Synthesis, antimicrobial evaluation and molecular modeling of 5-hydroxyisoquinolinium salt series; the effect of the hydroxyl moiety. Bioorg Med Chem. 2016;24:841-848. doi:10.1016/j.bmc.2016.01.006

62. Soukup O, Benkova M, Dolezal R, et al. The wide-spectrum antimicrobial effect of novel N-alkyl monoquaternary ammonium salts and their mixtures; the QSAR study against bacteria. Eur J Med Chem. 2020;206:112584. doi:10.1016/j.ejmech.2020.112584

63. Dolezal R, Soukup O, Malinak D, et al. Towards understanding the mechanism of action of antibacterial N-alkyl-3-hydroxypyridinium salts: biological activities, molecular modeling and QSAR studies. Eur J Med Chem. 2016;121:699-711. doi:10.10 16/j.ejmech.2016.05.058

64. Gessner A, Lieske A, Paulke B, Muller R. Influence of surface charge density on protein adsorption on polymeric nanoparticles: analysis by two-dimensional electrophoresis. Eur J Pharm Biopharm. 2002;54:165-170. doi:10.1016/S0939-6411(02)000 81-4
65. Elci SG, Jiang Y, Yan B, et al. Surface charge controls the suborgan biodistributions of gold nanoparticles. ACS Nano. 2016;10:5536-5542. doi:10.1021/acsnano.6b02086

66. Zarska M, Sramek M, Novotny F, et al. Biological safety and tissue distribution of (16-mercaptohexadecyl)trimethylammonium bromide-modified cationic gold nanorods. Biomaterials. 2018;154:275-290. doi:10.1016/j.biomaterials.2017.10.044

67. Mosmann T. Rapid colorimetric assay for cellular growth and survival: application to proliferation and cytotoxicity assays. J Immunol Methods. 1983;65:55-63. doi:10.1016/0022-1759(83) 90303-4

68. Malinak D, Dolezal R, Marek J, et al. 6-Hydroxyquinolinium salts differing in the length of alkyl side-chain: synthesis and antimicrobial activity. Bioorg Med Chem Lett. 2014;24: 5238-5241. doi:10.1016/j.bmcl.2014.09.060

69. Alkilany AM, Thompson LB, Boulos SP, Sisco PN, Murphy CJ. Gold nanorods: their potential for photothermal therapeutics and drug delivery, tempered by the complexity of their biological interactions. Adv Drug Deliv Rev. 2011;64:190-199. doi:10.1016/j.addr.2011.03.005

70. Novotný F. ParticleRecognition, a mathematica GUI interface for analysis of complex shaped nanoparticles in micrographs. Comput Phys Commun. 2017;214:98-104. doi:10.1016/j. cpc.2016.10.007

71. Chon JWM, Bullen C, Zijlstra P, Gu M. Spectral encoding on gold nanorods doped in a silica sol-gel matrix and its application to high-density optical data storage. Adv Funct Mater. 2007;17:875-880. doi:10.1002/adfm.200600565

72. Anselmo AC, Mitragotri S. Nanoparticles in the clinic. Bioeng Transl Med. 2016;1:10-29. doi:10.1002/btm2.10003

73. Pramod K, Indrajit R. Applications of gold nanoparticles in clinical medicine. Int J Pharm Pharm Sci. 2016;8:9-16.

74. Libutti SK, Paciotti GF, Byrnes AA, et al. Phase I and pharmacokinetic studies of CYT-6091, a novel PEGylated colloidal gold-rhTNF nanomedicine. Clin Cancer Res. 2010;16:61 39-6149. doi:10.1158/1078-0432.CCR-10-0978

75. Gandra N, Portz C, Nergiz SZ, Fales A, Vo-Dinh T, Singamaneni S. Inherently stealthy and highly tumor-selective gold nanoraspberries for photothermal cancer therapy. Sci Rep. 2015;5:10311. doi:10.1038/srep10311

76. Catone D, Ciavardini A, Di Mario L, et al. Plasmon controlled shaping of metal nanoparticle aggregates by femtosecond laser-induced melting. J Phys Chem Lett. 2018;9:5002-5008. doi:10.1021/acs.jpclett.8b02117

77. Johnson PS, Goel M, Abbott NL, Himpsel FJ. Helical versus all-trans conformations of oligo(ethylene glycol)-terminated alkanethiol self-assembled monolayers. Langmuir. 2014;30:102 63-10269. doi:10.1021/la500978s

78. Zorn S, Martin N, Gerlach A, Schreiber F. Real-time PMIRRAS studies of in situ growth of C11Eg6OMe on gold and immersion effects. Phys Chem Chem Phys. 2010;12:8985-8990. doi:10.10 39/b923691k

79. Schulz F, Vossmeyer T, Bastus NG, Weller H. Effect of the spacer structure on the stability of gold nanoparticles functionalized with monodentate thiolated poly(ethylene glycol) ligands. Langmuir. 2013;29:9897-9908. doi:10.1021/la401956c

80. Schulz F, Dahl GT, Besztejan S, et al. Ligand layer engineering to control stability and interfacial properties of nanoparticles. Langmuir. 2016;32:7897-7907. doi:10.1021/acs.langmuir.6b01 704

81. Inkpen MS, Liu ZF, Li H, Campos LM, Neaton JB, Venkataraman L. Non-chemisorbed gold-sulfur binding prevails in self-assembled monolayers. Nat Chem. 2019;11:351-358. doi:10.1038/s41557-019-0216-y

82. Pacchioni G. A not-so-strong bond. Nat Rev Mater. 2019;4:226. doi:10.1038/s41578-019-0094-3 
83. Galati E, Tao H, Rossner C, Zhulina EB, Kumacheva E. Morphological transitions in patchy nanoparticles. ACS Nano. 2020;14:4577-4584. doi:10.1021/acsnano.0c00108

84. Wijaya A, Schaffer SB, Pallares IG, Hamad-Schifferli K. Selective release of multiple DNA oligonucleotides from gold nanorods. ACS Nano. 2009;3:80-86. doi:10.1021/nn800702n

85. Braun GB, Pallaoro A, Wu G, et al. Laser-activated gene silencing via gold nanoshell-siRNA conjugates. ACS Nano. 2009;3:2007-2015. doi:10.1021/nn900469q

86. Inacio AS, Costa GN, Domingues NS, et al. Mitochondrial dysfunction is the focus of quaternary ammonium surfactant toxicity to mammalian epithelial cells. Antimicrob Agents Chemother. 2013;57:2631-2639. doi:10.1128/AAC.02437-12

87. Nagamune H, Maeda T, Ohkura K, Yamamoto K, Nakajima M, Kourai H. Evaluation of the cytotoxic effects of bis-quaternary ammonium antimicrobial reagents on human cells. Toxicol in Vitro. 2000;14:139-147. doi:10.1016/S0887-2333(00)00003-5

88. Kheir MM, Wang Y, Hua L, et al. Acute toxicity of berberine and its correlation with the blood concentration in mice. Food Chem Toxicol. 2010;48:1105-1110. doi:10.1016/j.fct.2010.01.033

89. Ohnuma A, Yoshida T, Tajima $H$, et al. Didecyldimethylammonium chloride induces pulmonary inflammation and fibrosis in mice. Exp Toxicol Pathol. 2010;62:643-651. doi:10.1016/j.etp.2009.08.007

90. Zhang S, Ding S, Yu J, Chen X, Lei Q, Fang W. Antibacterial activity, in vitro cytotoxicity, and cell cycle arrest of gemini quaternary ammonium surfactants. Langmuir. 2015;31:12161-12169. doi:10.1021/acs.langmuir.5b01430

91. Docherty KM, Kulpa JCF. Toxicity and antimicrobial activity of imidazolium and pyridinium ionic liquids. Green Chem. 2005;7:185-189. doi:10.1039/b419172b

92. Moore TL, Rodriguez-Lorenzo L, Hirsch V, et al. Nanoparticle colloidal stability in cell culture media and impact on cellular interactions. Chem Soc Rev. 2015;44:6287-6305. doi:10.1039/ C4CS00487F

93. Muller KH, Motskin M, Philpott AJ, et al. The effect of particle agglomeration on the formation of a surface-connected compartment induced by hydroxyapatite nanoparticles in human monocyte-derived macrophages. Biomaterials. 2014;35:10 74-1088. doi:10.1016/j.biomaterials.2013.10.041

94. Liu X, Huang N, Li H, Jin Q, Ji J. Surface and size effects on cell interaction of gold nanoparticles with both phagocytic and nonphagocytic cells. Langmuir. 2013;29:9138-9148. doi:10.1021/ la401556k

95. Pamies R, Cifre JGH, Espín VF, Collado-González M, Fgd B, de la Torre JG. Aggregation behaviour of gold nanoparticles in saline aqueous media. J Nanopart Res. 2014;16:2376. doi:10.1007/ s11051-014-2376-4

96. Tsai DH, Davila-Morris M, DelRio FW, Guha S, Zachariah MR, Hackley VA. Quantitative determination of competitive molecular adsorption on gold nanoparticles using attenuated total reflectance-Fourier transform infrared spectroscopy. Langmuir. 2011;27:9302-9313. doi:10.1021/la2005425

97. Heinz H, Pramanik C, Heinz O, et al. Nanoparticle decoration with surfactants: molecular interactions, assembly, and applications. Surf Sci Rep. 2017;72:1-58.

98. Hohenstein EG, Sherrill CD. Effects of heteroatoms on aromatic pi-pi interactions: benzene-pyridine and pyridine dimer. $J$ Phys Chem A. 2009;113:878-886. doi:10.1021/jp809062x
99. Mecozzi S, West AP, Dougherty DA. Cation-pi interactions in aromatics of biological and medicinal interest: electrostatic potential surfaces as a useful qualitative guide. Proc Natl Acad Sci U S A. 1996;93:10566-10571. doi:10.1073/pnas.93.20.10566

100. Wu M, Vartanian AM, Chong GN, et al. Solution NMR analysis of ligand environment in quaternary ammonium-terminated self -assembled monolayers on gold nanoparticles: the effect of surface curvature and ligand structure. $J$ Am Chem Soc. 2019;141:4316-4327. doi:10.1021/jacs.8b11445

101. Lorenz S, Hauser CP, Autenrieth B, Weiss CK, Landfester K, Mailander V. The softer and more hydrophobic the better: influence of the side chain of polymethacrylate nanoparticles for cellular uptake. Macromol Biosci. 2010;10:1034-1042. doi:10.1002/mabi.201000099

102. Dausend J, Musyanovych A, Dass M, et al. Uptake mechanism of oppositely charged fluorescent nanoparticles in HeLa cells. Macromol Biosci. 2008;8:1135-1143. doi:10.1002/ mabi.200800123

103. Kennedy LC, Bickford LR, Lewinski NA, et al. A new era for cancer treatment: gold-nanoparticle-mediated thermal therapies. Small. 2011;7:169-183. doi:10.1002/smll.201000134

104. Ali MR, Rahman MA, Wu Y, et al. Efficacy, long-term toxicity, and mechanistic studies of gold nanorods photothermal therapy of cancer in xenograft mice. Proc Natl Acad Sci U S A. 2017;114: E3110-e8. doi:10.1073/pnas.1619302114

105. Majumdar S, Sierra-Suarez JA, Schiffres SN, et al. Vibrational mismatch of metal leads controls thermal conductance of self-assembled monolayer junctions. Nano Lett. 2015;15:2985-2991. doi:10.1021/n1504844d

106. Luo T, Lloyd J. Non-equilibrium molecular dynamics study of thermal energy transport in Au-SAM-Au junctions. Int $J$ Heat Mass Transf. 2010;53:1-11. doi:10.1016/j.ijheatmasstransfer.20 09.10.033

107. Park J, Huang J, Wang W, Murphy CJ, Cahill DG. Heat transport between $\mathrm{Au}$ nanorods, surrounding liquids, and solid supports. J Phys Chem C. 2012;116:26335-26341. doi:10.1021/jp308130d

108. Schmidt AJ, Alper JD, Chiesa M, Chen G, Das SK, HamadSchifferli K. Probing the gold nanorod-ligand-solvent interface by plasmonic absorption and thermal decay. J Phys Chem C. 2008;112:13320-13323. doi:10.1021/jp8051888

109. Liu Y, Sun W, Wang K, Xu -J-J, Chen H-Y, Xia X-H. End group properties of thiols affecting the self-assembly mechanism at gold nanoparticles film as evidenced by water infrared probe. Anal Chem. 2019;91:14508-14513. doi:10.1021/acs.analchem.9b03 332

110. Gu ZT, Wang H, Li L, et al. Heat stress induces apoptosis through transcription-independent p53-mediated mitochondrial pathways in human umbilical vein endothelial cell. Sci Rep. 2014;4. doi:10.1038/srep04469

111. Ratto F, Matteini P, Rossi F, et al. Photothermal effects in connective tissues mediated by laser-activated gold nanorods. Nanomed-Nanotechnol. 2009;5:143-151. doi:10.1016/j.nano.20 08.10 .002

112. Lukianova-Hleb EY, Koneva II, Oginsky AO, La Francesca S, Lapotko DO. Selective and self-guided micro-ablation of tissue with plasmonic nanobubbles. J Surg Res. 2011;166:E3-E13. doi:10.1016/j.jss.2010.10.039 


\section{Publish your work in this journal}

The International Journal of Nanomedicine is an international, peerreviewed journal focusing on the application of nanotechnology in diagnostics, therapeutics, and drug delivery systems throughout the biomedical field. This journal is indexed on PubMed Central, MedLine, CAS, SciSearch ${ }^{\mathbb{B}}$, Current Contents ${ }^{\mathbb{B}} /$ Clinical Medicine, $^{2}$
Journal Citation Reports/Science Edition, EMBase, Scopus and the Elsevier Bibliographic databases. The manuscript management system is completely online and includes a very quick and fair peer-review system, which is all easy to use. Visit http://www.dovepress.com/ testimonials.php to read real quotes from published authors. 